\title{
Tutorial on photoacoustic tomography
}

\author{
Yong Zhou \\ Junjie Yao \\ Lihong V. Wang
}




\title{
Tutorial on photoacoustic tomography
}

\author{
Yong Zhou, Junjie Yao, and Lihong V. Wang*
}

Washington University in St. Louis, Department of Biomedical Engineering, Optical Imaging Laboratory, One Brookings Drive, Campus Box 1097, St. Louis, Missouri 63130, United States

\begin{abstract}
Photoacoustic tomography (PAT) has become one of the fastest growing fields in biomedical optics. Unlike pure optical imaging, such as confocal microscopy and two-photon microscopy, PAT employs acoustic detection to image optical absorption contrast with high-resolution deep into scattering tissue. So far, PAT has been widely used for multiscale anatomical, functional, and molecular imaging of biological tissues. We focus on PAT's basic principles, major implementations, imaging contrasts, and recent applications. ๑ 2016 Society of PhotoOptical Instrumentation Engineers (SPIE) [DOI: 10.1117/1.JBO.21.6.061007]
\end{abstract}

Keywords: Photoacoustic tomography; photoacoustic computed tomography; photoacoustic microscopy; optical absorption contrast. Paper 160054SSTR received Jan. 26, 2016; accepted for publication Mar. 22, 2016; published online Apr. 18, 2016.

\section{Introduction}

Photoacoustic tomography (PAT), also called optoacoustic tomography, is a three-dimensional (3-D) imaging modality based on the photoacoustic (PA) effect. Although the PA effect was discovered more than a century ago by Alexander Graham Bell, it has found applications in biomedical imaging only in the last decade or so. Nowadays, PAT is one of the largest research areas in biomedical optics and is still growing rapidly. ${ }^{1}$

Harnessing both rich optical absorption contrast and high ultrasonic resolution, PAT is a hybrid imaging modality that can image deep tissues. While pure optical imaging modalities can also detect optical absorption by monitoring intensity variations in transmitted or reflected light, their sensitivities are usually two orders of magnitude lower than that of PAT. ${ }^{2}$ In addition, because acoustic waves scatter much more weakly than light in biological tissues, they can propagate a greater distance than photons without losing their original propagation directions, providing PAT with high spatial resolution at depths. While pure ultrasonic imaging can also achieve high spatial resolution in deep tissues, its mechanical contrast is incapable of providing certain physiological parameters, such as the oxygen saturation of hemoglobin and the metabolic rate of oxygen.

Several comprehensive reviews of PAT can be found in the literature $;^{1,3-21}$ some are general, ${ }^{1,3-5,11,21}$ and some focus on specific areas, such as PAT's application in imaging molecules, ${ }^{9}$ microvasculature, ${ }^{6}$ tumors, ${ }^{17}$ the brain, ${ }^{16}$ and small animals. ${ }^{15}$ Here, we will review the fundaments of PAT, including its principles, major implementations, system characteristics, main contrast agents, and recent applications.

\section{Principles}

\subsection{Initial Pressure Rise}

In PAT, a short-pulsed light source is typically used to irradiate the tissue, resulting in broadband PA waves with a frequency content extending to several tens or even hundreds of megahertz for acoustic detection. Following absorption of the light, an

\footnotetext{
*Address all correspondence to: Lihong V. Wang, E-mail: Ihwang@wustl.edu
}

initial temperature rise induces a pressure rise, which propagates as a photoacoustic wave and finally is detected by a singleelement ultrasonic transducer or a transducer array. There are two important time scales in the generation of PA waves, ${ }^{2}$ the thermal relaxation time $\left(\tau_{\text {th }}\right)$ and the stress relaxation time $\left(\tau_{s}\right) . \tau_{\text {th }}$ denotes the thermal relaxation (thermal diffusion) time of the desired voxel, and is given by

$\tau_{\text {th }}=\frac{d_{c}^{2}}{\alpha_{\text {th }}}$,

where $d_{c}$ is the desired spatial resolution and $\alpha_{\mathrm{th}}$ is the thermal diffusivity $\left(\mathrm{m}^{2} / \mathrm{s}\right) . \tau_{s}$, which characterizes the stress relaxation time of the desired voxel, is given by

$\tau_{s}=\frac{d_{c}}{v_{s}}$,

where $v_{s}$ is the speed of sound $(\mathrm{m} / \mathrm{s})$.

Upon laser excitation, the fractional volume expansion of the heated region $\mathrm{d} V / V$ can be expressed as

$\frac{\mathrm{d} V}{V}=-\kappa p+\beta T$,

where $\kappa$ denotes the isothermal compressibility $\left(\mathrm{Pa}^{-1}\right), p$ denotes the change in pressure $(\mathrm{Pa}), \beta$ denotes the thermal coefficient of volume expansion $\left(\mathrm{K}^{-1}\right)$, and $T$ denotes the change in temperature $(\mathrm{K})$.

If the laser pulse duration is shorter than $\tau_{\text {th }}$ and $\tau_{s}$, the excitation satisfies both thermal and stress confinements. In this situation, the fractional volume change is negligible. Thus, the initial pressure rise $p_{0}$ can be derived from

$p_{0}=\frac{\beta T}{\kappa}$.

Further, the local temperature rise can be expressed as

$$
T=\frac{\eta_{\mathrm{th}} A_{e}}{\rho C_{V}}
$$


where $\eta_{\text {th }}$ is the percentage of absorbed light converted into heat, and $A_{e}$ is the specific optical energy deposition $\left(\mathrm{J} / \mathrm{m}^{3}\right)$. Substituting Eq. (5) into Eq. (4), we have

$\mathrm{p}_{0}=\frac{\beta}{\kappa \rho \mathrm{C}_{\mathrm{V}}} \eta_{\mathrm{th}} \mathrm{A}_{\mathrm{e}}$

By defining the Gruneisen parameter $\Gamma$ (dimensionless) as

$$
\Gamma=\frac{\beta}{\kappa \rho C_{V}},
$$

$$
\text { Equation (6) becomes }
$$

$p_{0}=\Gamma \eta_{\mathrm{th}} A_{e}$.

For single-photon optical absorption, $A_{e}$ is proportional to the local optical fluence $F\left(\mathrm{~J} / \mathrm{cm}^{2}\right)$. In this case, Eq. (8) becomes

$$
p_{0}=\Gamma \eta_{\mathrm{th}} \mu_{a} F
$$

where $\mu_{a}$ is the optical absorption coefficient $\left(\mathrm{cm}^{-1}\right)$. Based on Eq. (9), the initial pressure rise is proportional to $\mu_{a}$ and $F$. $\Gamma$ and $\eta_{\text {th }}$ are usually approximated as constants, although they have been found to depend on the tissue type; ${ }^{22,23}$ thus, if $p_{0}$ can be measured and $F$ is known, $\mu_{a}$ can be recovered. After the generation of the initial pressure $p_{0}$, an acoustic wave starts to propagate at the speed of sound in the material. The propagation in an inviscid medium can be described by general photoacoustic equations in the time-domain, as discussed in Sec. 2.2.

\subsection{Photoacoustic Wave Propagation}

The propagation and generation of acoustic pressure $p(\vec{r}, t)$ at position $\vec{r}$ and time $\mathrm{t}$ is governed by the following wave equation:

$$
\left(\nabla^{2}-\frac{1}{v_{s}^{2}} \frac{\partial^{2}}{\partial t^{2}}\right) p(\vec{r}, t)=-\frac{\beta}{\kappa v_{s}^{2}} \frac{\partial^{2} T(\vec{r}, t)}{\partial t^{2}} .
$$

Note that $T$ represents the temperature rise instead of the temperature, i.e., the temperature rise above its initial value. Under the condition of thermal confinement, where heat conduction is negligible, the heat diffusion equation becomes

$\rho \mathrm{C}_{\mathrm{V}} \frac{\partial T(\vec{r}, t)}{\partial t}=H(\vec{r}, t)$,

where $H$ is the heating function, defined as the thermal energy deposited per unit volume and per unit time. Note that the heating function $H$ is related to the specific optical energy deposition $A_{e}$ by the following equation:

$\mathrm{H}(\vec{r}, t)=\eta_{\mathrm{th}} \frac{\partial A_{e}(\vec{r}, t)}{\partial t}$.

Substituting Eq. (11) into Eq. (10), we have

$$
\left(\nabla^{2}-\frac{1}{v_{s}^{2}} \frac{\partial^{2}}{\partial t^{2}}\right) p(\vec{r}, t)=-\frac{\beta}{C_{P}} \frac{\partial H(\vec{r}, t)}{\partial t}
$$

where $C_{P}$ is the specific heat capacity at constant pressure.
Solving Eq. (13) with the Green function approach, we have the following delta heating response:

$p_{\delta}(\vec{r}, t)=\frac{1}{4 \pi v_{s}^{2}} \frac{\partial}{\partial t}\left[\int \mathrm{d} \vec{r}^{\prime} \frac{p_{0}\left(\vec{r}^{\prime}\right)}{\left|\vec{r}-\vec{r}^{\prime}\right|} \delta\left(t-\frac{\left|\vec{r}-\vec{r}^{\prime}\right|}{v_{s}}\right)\right]$,

where $p_{0}\left(\vec{r}^{\prime}\right)$ is the initial pressure rise at location $\vec{r}^{\prime}$. If the heating pulse has a finite duration, the response can be computed by convolution

$p(\vec{r}, t)=\int_{-\infty}^{+\infty} \mathrm{d} t^{\prime} p_{\delta}\left(\vec{r}, t-t^{\prime}\right) S\left(t^{\prime}\right)$

where $S(t)$ is the temporal profile of the excitation pulse.

\section{Photoacoustic Image Formation}

The goal of PA imaging is to retrieve the local pressure rise $p_{0}$ inside the tissue. Based on Eq. (9), if we know the local optical fluence $F$, the absorption coefficient $\mu_{a}$ can then be calculated. In practice, the adjacent fluence $F$ in the tissue is usually comparable, but the absorption coefficient $\mu_{a}$ differs considerably. For example, blood in the visible light region has much stronger absorption than other components in tissue. ${ }^{14}$ Thus, if $F$ is assumed to be regionally homogeneous in anatomic PA imaging, then $p_{0}$ can be used to directly map the relative absorption coefficient $\mu_{a}$. There are two basic methods to recover the original $p_{0}$ distribution inside the target once the pressures at the observation points are measured: ${ }^{11}$ reconstruction-based image formation and focused-scanning image formation. While the former is the basis of photoacoustic computed tomography (PACT), the latter is commonly used for photoacoustic microscopy (PAM) and occasionally for photoacoustic macroscopy (PAMac). Because PAM and PAMac mainly differ in their spatial resolution, i.e., PAM has a spatial resolution less than $50 \mu \mathrm{m}$ while PAMac does not, we will only discuss PAM in this review paper.

\subsection{Image Formation in Photoacoustic Computed Tomography}

For PACT, the light is expanded to illuminate the whole object to be imaged. PA signals are acquired at multiple locations around the object, either by using a transducer array or by scanning a single-element transducer to simulate an array. Next, back-projecting all the PA data, similar to traditional computed tomography or positron emission tomography imaging, generates PA images of the object. Note that in order to detect PA signals from the same object at multiple locations, a transducer or transducer array with a large acceptance angle is desirable. Several methods are widely used for PA image formation, ${ }^{24,25-30}$ such as universal back-projection (UBP) ${ }^{25}$ and time reversal. ${ }^{26}$

Demonstrated in spherical, cylindrical, and planar detection geometries, the UBP algorithm has the following formula:

$$
p_{0}\left(\vec{r}^{\prime}\right)=\frac{1}{\Omega_{0}} \int_{S} \mathrm{~d} \Omega_{0} \times\left. 2\left[p(\vec{r}, t)-t \frac{\partial p(\vec{r}, t)}{\partial t}\right]\right|_{v_{s} t=\left|\vec{r}-\vec{r}^{\prime}\right|}
$$

where $\Omega_{0}$ is the solid angle of the entire detection surface $S$ with respect to a source point at $\vec{r}^{\prime}$. The factor $d \Omega_{0} / \Omega_{0}$ weighs the contribution from each element on the detection surface $S$. The 
term $p(\vec{r}, t)$ is the direct back-projection of the detected PA signals onto a spherical surface centered at $\vec{r}$. The first derivative with respect to time represents a ramp filter, which suppresses low frequency signals. In the UBP algorithm, the medium is assumed to be acoustically lossless and homogeneous.

The time-reversal method has recently been recognized as the least restrictive reconstruction algorithm: it can work for any closed geometry and can incorporate acoustic heterogeneities. In the time-reversal method, the measured acoustic pressure is retransmitted into the medium in time reversed order. The same wave equation is solved from $t=T$ to $t=0$, where $T$ is the maximum time for the acoustic wave to traverse the detection region. The measured pressure data are treated either as boundary conditions or as a source. To solve the wave equation, numerical methods are employed, such as time-domain finite-difference techniques or k-space pseudospectral methods. Thus, the time-reversal method is more computationally intensive than the UBP method, especially for 3-D image reconstruction.

\subsection{Image Formation in Photoacoustic Microscopy}

Different from reconstruction-based methods, the focused-scanning scheme of PAM usually focuses both the optical excitation and acoustic detection. If the optical focus is tighter than the acoustic focus, the technique is called optical resolution photoacoustic microscopy (OR-PAM); ${ }^{31,32}$ otherwise, it is called acoustic resolution photoacoustic microscopy (AR-PAM). ${ }^{33-35}$ In both cases, each laser pulse generates a one-dimensional (1-D) photoacoustic image (A-line) along the axial direction. Raster-scanning laterally and then piecing together all the Alines provides a 3-D PA image. Because each signal acquired by the transducer directly represents a 1-D image of a single line inside the object after minimal signal processing, there is no need for image reconstruction. Although raster-scanning is used in most cases, there are alternative scanning methods, such as circumferential-section-scanning for endoscopic imaging, ${ }^{36,37-40} 3$-D arbitrary scanning for blood vessel monitoring, ${ }^{41}$ and random access scanning for cell tracking. ${ }^{42}$

\section{Photoacoustic Systems and Their Characteristics}

As mentioned earlier, based on their different image formation mechanism, PA systems can be classified as either reconstructionbased PACT or focused-scanning-based PAM. In this section, we discuss typical PA systems and their characteristics, including spatial resolution, imaging speed, and penetration depth.

\subsection{Photoacoustic Computed Tomography}

Current PACT systems use spherical, ${ }^{43,44-46}$ cylindrical, ${ }^{15,47-52}$ or planar detection geometry. ${ }^{53-60}$ Each geometry has several implementations. For spherical-view systems, either an arcshaped transducer array ${ }^{44}$ or a hemispherical array with a spiral pattern ${ }^{45,46}$ is used. In both cases, mechanical scanning is required for dense spatial sampling, and 3-D reconstruction is performed. Figure 1(a) shows a hemispherical array based PACT system, ${ }^{46}$ with 512 ultrasonic transducer elements distributed in a hemispherical shell with a radius of $127 \mathrm{~mm}$. The diameter of each transducer element is $3 \mathrm{~mm}$, and the center frequency is $2 \mathrm{MHz}$, with a $70 \%$ bandwidth. The transducer array is scanned spirally to achieve dense spatial sampling for image reconstruction. As shown in Fig. 1(a), the bottom of the hemispherical shell contains an aperture for light delivery. In this case, pulses from an Alexandrite laser at $756 \mathrm{~nm}$ are used to excite the target. Depending on the spiral scanning pattern, total data acquisition time varies from $12 \mathrm{~s}$ for the smallest spiral (24-mm radius) to $3.2 \mathrm{~min}$ for the largest spiral (96-mm radius). Using a thin graphite fiber phantom (6- $\mu \mathrm{m}$ diameter), the resolution of this system was quantified to be $0.42 \mathrm{~mm}$. Because spherical-view detection was implemented, this resolution was constant at different graphite fiber orientations. A $5.3-\mathrm{cm}$ penetration was achieved in the phantom. As shown in Fig. 1(b), blood vessels in the breasts of two healthy volunteers were clearly imaged although their depths are not shown.

A representative cylindrical-view PACT system $^{48}$ is shown in Fig. 2. The system contains a 512-element full ring transducer array with a ring diameter of $5 \mathrm{~cm}$. Each transducer element has a center frequency of $5 \mathrm{MHz}$ and an $80 \%$ (one-way) detection
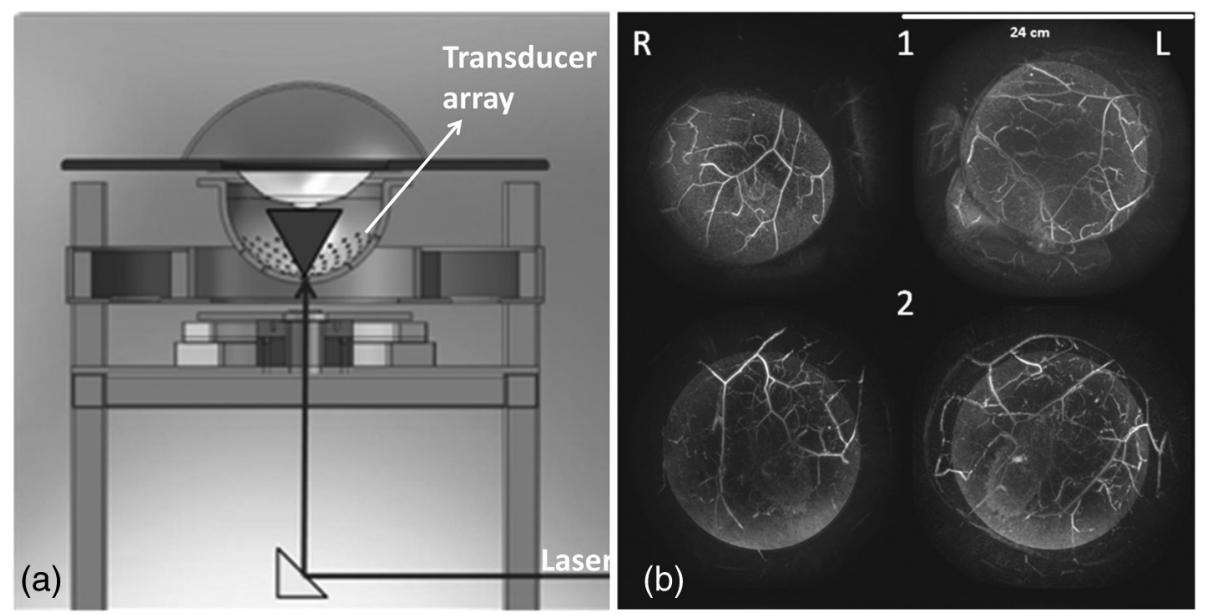

Fig. 1 Hemispherical array based photoacoustic computed tomography (PACT) system and its representative images. (a) Schematic of a spherical-view photoacoustic system. (b) Representative human breast images from two healthy volunteers (1) and (2). R, right breast and L, left breast. Reproduced with permission from Refs. 45 and 46. 

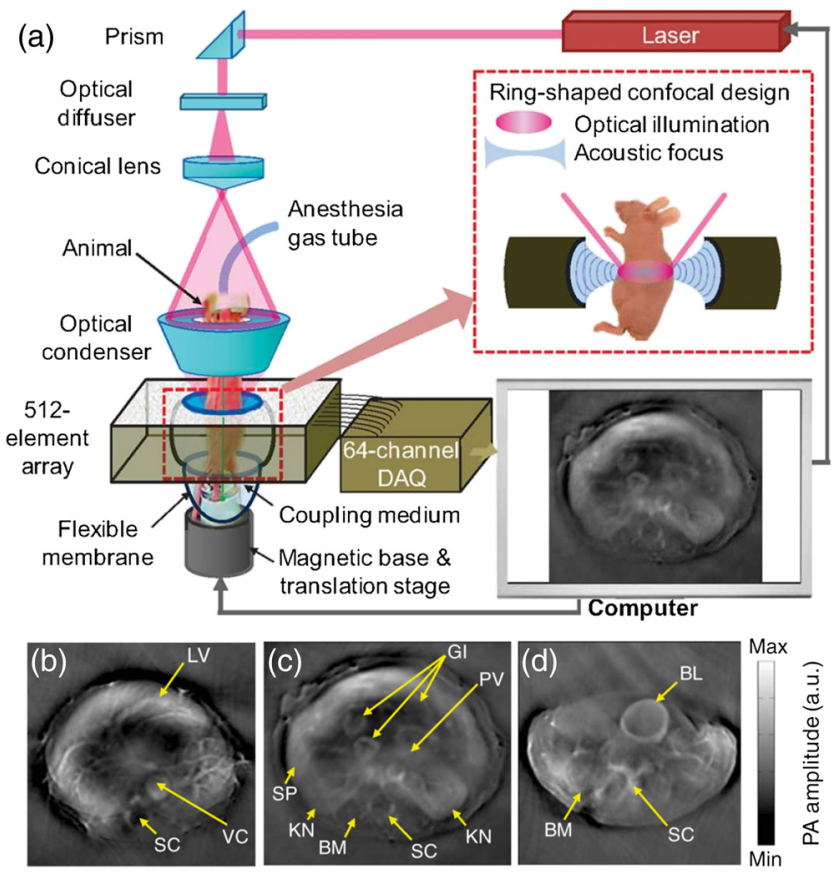

Fig. 2 Cylindrical-view PACT system and its representative images. (a) Schematic of the system, showing the confocal design of both the optics and acoustics. (b)-(d) In vivo images of athymic mice acquired by the system at different anatomical locations: (b) liver, (c) kidneys, and (d) bladder. BL, bladder; BM, backbone muscle; Gl, GI tract; KN, kidney; LV, liver; PV, portal vein; SC, spinal cord; SP, spleen; and VC, vena cava. Reproduced with permission from Ref. 48.

bandwidth. ${ }^{61}$ To improve the cross-sectional imaging ability, each element is cylindrically focused to reject out-of-plane signals. The combined foci from all elements provide a central imaging region with a $\sim 20 \mathrm{~mm}$ diameter and $\sim 1$-mm thickness. Strictly speaking, such a system is a circular-view system, since only a ring is used to reconstruct the image instead of a cylinder. However, by taking advantage of its cylindrical focusing capability, high-quality two-dimensional (2-D) cross-sectional images are attainable. In addition, by scanning the sample or the array along the elevational direction, 3-D images can be acquired. Within the imaging region, the system provides 100 - to $250-\mu \mathrm{m}$ transverse resolution in the circumferential direction and $100-\mu \mathrm{m}$ axial resolution in the radial direction. Limited by its 64 -channel data acquisition and $10-\mathrm{Hz}$ laser, the system acquires one frame/1.25 s. However, by employing a 512-channel real-time data acquisition system and a faster laser, higher rate imaging can be realized. Because of the fixed ring diameter, only $1-\mathrm{cm}$ penetration depth was reported. As shown in Figs. 2(b) and 2(c), blood-rich organs, such as the liver, kidneys, spleen, spine, and GI tracts can be clearly visualized. In addition, blood-poor organs, such as the bladder, can also be imaged with the help of a near-infrared contrast agent (IRDye800, LI-COR, Inc.), as shown in Fig. 2(d).

There are different implementations for planar-view PACT, using either a 2-D ultrasound transducer array ${ }^{44,53}$ or a Fabry-Perot interferometer (FPI). ${ }^{55,60}$ Generally, the 2-D transducer array based PACT system has a higher frame rate, while the FPI-based system has higher sensitivity and a larger receiving angle. Figure 3(a) is a schematic of the FPI-based PACT system. ${ }^{62}$ In this type of PA imaging, the deformation of pressure-sensitive materials (e.g., polymer) is measured by optical resonance. The PA excitation beam was at $640 \mathrm{~nm}$, and the PA probing beam was at $1550 \mathrm{~nm}$. By raster scanning the probe beam across the FPI surface, photoacoustic waves can be mapped in 2-D. Depending on the detector bandwidth (22 $\mathrm{MHz}$ in this work), the axial resolution of this system was $27 \mu \mathrm{m}$. The lateral resolution was about $120 \mu \mathrm{m}$, which was determined by primarily the detection bandwidth and angular range. To scan an area of $16 \times 16 \mathrm{~mm}^{2}$, the image acquisition time was about $8 \mathrm{~min}$, which was limited by the $50-\mathrm{Hz}$ pulse repetition rate of the excitation laser. The in vivo penetration depth of this system was demonstrated to be more than $10 \mathrm{~mm}$. As shown in Fig. 3(b), two embryos (shaded in red) with detailed structures, such as the liver, ribs, pulmonary vein, and right atrium, can be clearly imaged.

\subsection{Photoacoustic Microscopy}

As mentioned earlier, there are two types of PAM: OR-PAM and AR-PAM. In OR-PAM, although the optical focus determines its lateral resolution, the detection transducer is also placed confocally with the optical focus to maximize the system's detection sensitivity. Similarly, in AR-PAM, the excitation beam fills the entire acoustic focus to maximize the system's sensitivity. The axial resolution in both OR-PAM and AR-PAM is determined
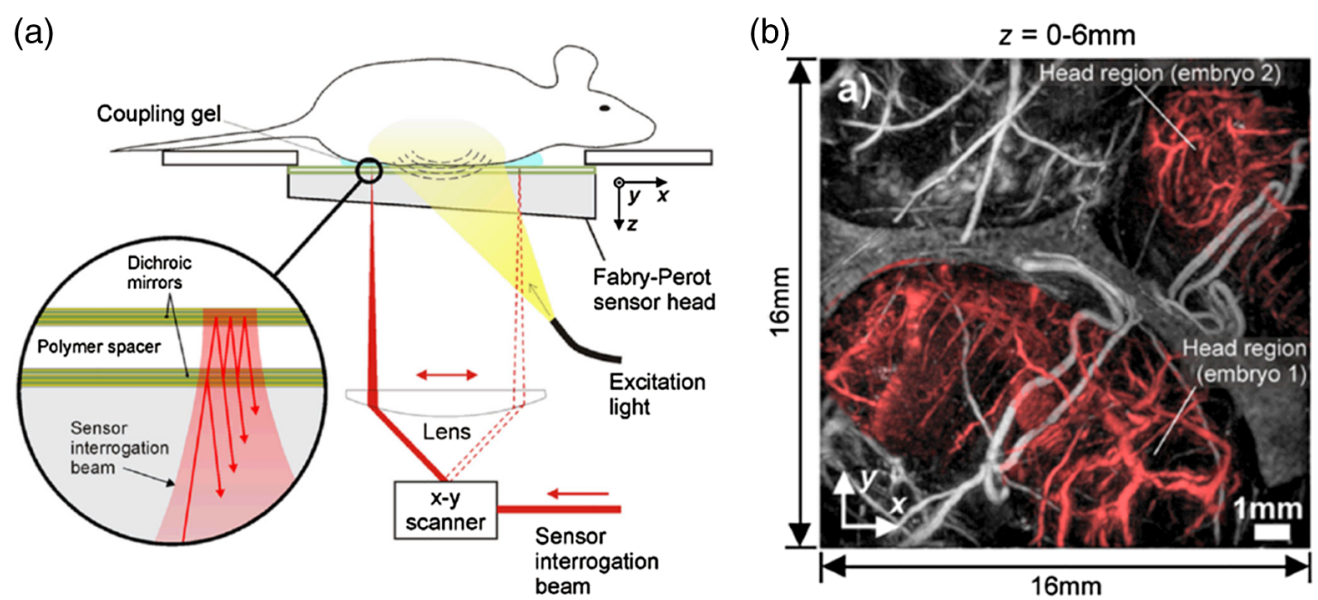

Fig. 3 Fabry-Perot interferometer (FBI) based PACT system. (a) Schematic and (b) a representative image of a FBI based PACT system. The red parts in (b) indicate the location of embryos. Reproduced with permission from Ref. 62. 


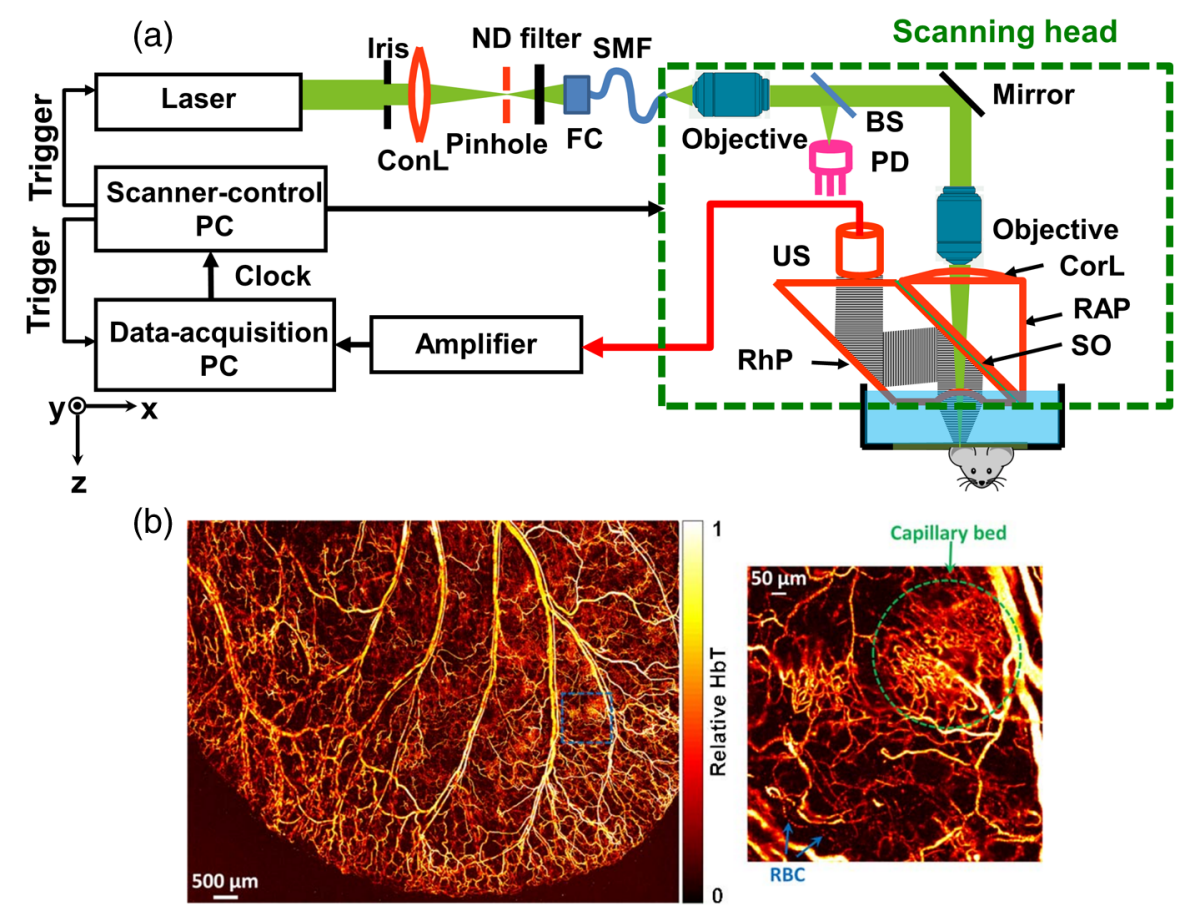

Fig. 4 Typical optical resolution photoacoustic microscopy (OR-PAM) system. (a) Schematic and (b) a representative image of OR-PAM. BS, beam splitter; ConL, condenser lens; CorL, correction lens; FC, fiber collimator; $\mathrm{HbT}$, total hemoglobin concentration; ND, neutral density; $\mathrm{PD}$, photodiode; RAP, right-angle prism; RBC, red blood cell; RhP, rhomboid prism; SMF, single-mode fiber; SO, silicone oil; and US, ultrasonic transducer. Reproduced with permission from Ref. 31.

by the detection bandwidth of the transducer. Due to the frequency dependence of acoustic attenuation, the bandwidth is chosen according to the desired imaging depth.

As shown in Fig. 4(a), a typical OR-PAM system employs an optical lens to focus light into the sample. A light-sound combiner transmits the light and reflects the sound. The combiner is composed of a right-angled prism, a thin layer of silicone oil, and a rhomboid prism for acoustic-optical coaxial alignment. Usually the laser beam is tightly focused, whose diameter can range from several hundred nanometers to several micrometers, depending on the numerical aperture (NA) of the optical focusing lens, the wavelength of the excitation beam, and the desired imaging depth. Relying on the tight optical focus, the penetration of an OR-PAM system is limited to about one transport mean free path in tissue $(\sim 1 \mathrm{~mm}) .{ }^{12,63,64}$ However, by using longer wavelength laser pulses, which have longer transport mean free paths, the penetration limit can be increased. ${ }^{65}$ OR-PAM can image vasculature in a mouse ear, ${ }^{31}$ eye, ${ }^{66-69}$ and brain $^{31,70,71}$ clearly. Figure 4(b) shows a representative mouse ear image acquired with OR-PAM, where both a capillary bed and flowing red blood cells can be clearly visualized.

As shown in Fig. 5(a), in a typical AR-PAM system, the laser beam passes through a conical lens to form a ring-shaped illumination pattern. ${ }^{34,72,73-75}$ The beam is then focused into the target by custom-made mirrors. The optical illumination on the skin surface has a donut shape with a dark center to minimize strong surface signals. Since acoustic scattering is much weaker than optical scattering in tissue, tight acoustic focusing can be maintained at depths. For example, using a 50-MHz center frequency transducer with an NA of 0.44 , a lateral resolution of $\sim 45 \mu \mathrm{m}$ was achieved with an imaging depth of more than $3 \mathrm{~mm}$. By choosing transducers with different center frequencies and NAs, the lateral resolutions can be scaled. Note that although acoustic scattering is weak in tissue, high frequency ultrasound signals suffer strong attenuation. In the end, the attenuation becomes the limiting factor for deep high-resolution AR-PAM imaging. Figure 5(b) shows a representative AR-PAM image of a human forearm, where detailed vasculatures can be

(a)

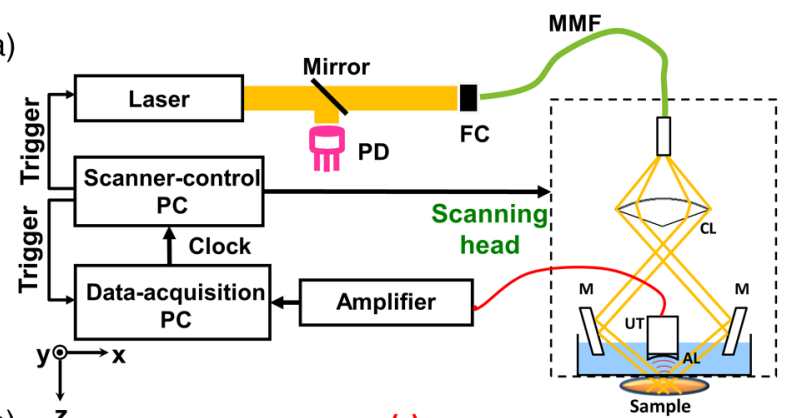

(b)

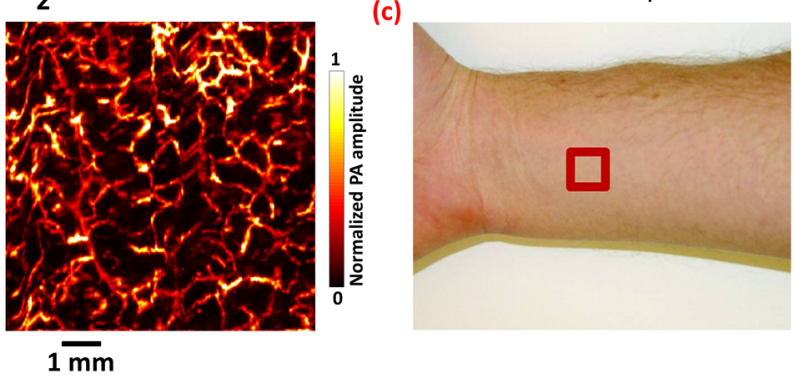

Fig. 5 Typical acoustic resolution photoacoustic microscopy (ARPAM) system. (a) Schematic and (b) a representative image of AR-PAM. AL, acoustic lens; CL, conical lens; FC, fiber coupler; M, mirror; MMF, multimode fiber; PD, photodiode; and UT, ultrasonic transducer. (c) Red box is the image area. Reproduced with permission from Ref. 74. 
Table 1 Summary of typical PA system characteristics.

\begin{tabular}{|c|c|c|c|}
\hline Modality & $\begin{array}{l}\text { Lateral } \\
\text { resolution } \\
(\mu \mathrm{m})\end{array}$ & $\begin{array}{l}\text { Axial } \\
\text { resolution } \\
(\mu \mathrm{m})\end{array}$ & $\begin{array}{l}\text { Imaging } \\
\text { depth } \\
(\mathrm{mm})\end{array}$ \\
\hline Subwavelength OR-PAM ${ }^{76}$ & 0.22 & 15 & $0.1^{\mathrm{a}}$ \\
\hline Second generation OR-PAM ${ }^{31}$ & 2.5 & 15 & $1.2^{\mathrm{a}}$ \\
\hline Dark-field AR-PAM ${ }^{34}$ & 45 & 15 & $3^{\mathrm{a}}$ \\
\hline Bright-field AR-PAM ${ }^{77}$ & 44 & 15 & $4.8^{\mathrm{a}}$ \\
\hline Spherical-view $\mathrm{PACT}^{46}$ & 420 & 420 & $53^{b}$ \\
\hline Cylindrical-view $\mathrm{PACT}^{48}$ & $100-250$ & 100 & $10^{\mathrm{a}}$ \\
\hline FPI PACT ${ }^{62}$ & 120 & 27 & $10^{\mathrm{a}}$ \\
\hline Clinical linear array $P A C T^{11,78}$ & 720 & 640 & $70^{\mathrm{b}}$ \\
\hline
\end{tabular}

Note: FPI: Fabry-Perot interferometer.

a Based on in vivo data.

${ }^{\mathrm{b}}$ Based on phantom data.

clearly discerned. Figure 5(c) is a photo of the forearm, where the red box indicates the image area of $8 \mathrm{~mm} \times 8 \mathrm{~mm}$.

The lateral and axial resolutions as well as imaging depth are summarized in Table 1.

\section{Photoacoustic Contrast Agents}

Theoretically speaking, any material with sufficiently high optical absorption can be detected by PAT. Thus, by choosing the right wavelengths, PAT potentially can be used to image all materials. Specifically, in biological applications, absorbers are usually divided into endogenous and exogenous categories. We will discuss these two categories in Secs. 5.1 and 5.2.

\subsection{Endogenous Contrast Agents}

The primary advantage of endogenous contrast agents is that they allow label-free imaging, so they do not affect the original biological environment. In biological tissue, there are varieties of optical absorbers, ${ }^{14}$ such as DNA/RNA, ${ }^{79,80}$ cytochromes, ${ }^{81}$ bilirubin, ${ }^{82}$ myoglobin, ${ }^{83,84}$ hemoglobin, ${ }^{85,86}$ methemoglobin, ${ }^{87}$ carboxyhemoglobin, ${ }^{88}$ melanin, ${ }^{54,89,90}$ lipid, ${ }^{91-93}$ water, ${ }^{94,95}$ and glucose. ${ }^{96,97}$ Among all these agents, DNA/RNA is commonly used for cell nuclear imaging in the ultraviolet region [Fig. 6(a)], ${ }^{79}$ hemoglobin is widely used for vascular imaging in the visible and the near-infrared spectral regions [Fig. 6(b)], ${ }^{19}$ and melanin is employed for melanoma tumor imaging in the near-infrared region [Fig. 6(c) $].{ }^{54}$ In addition, in the nearinfrared region, lipids and water are used for atherosclerotic plaque $^{98,99}$ and injury ${ }^{94}$ imaging, respectively. As shown in Fig. 7, different endogenous contrast agents have different absorption spectra. Thus, PAT can separate them with spectral measurement, when the local optical fluence is known.

A representative application of spectral decomposition is to quantify the oxygen saturation of hemoglobin $\left(\mathrm{sO}_{2}\right)$ in blood vessels by differentiating signals from oxy-hemoglobin $\left(\mathrm{HbO}_{2}\right)$ and deoxy-hemoglobin (HbR). As derived above, the measured PA amplitude of blood is proportional to the local fluence and the absorption coefficient of blood

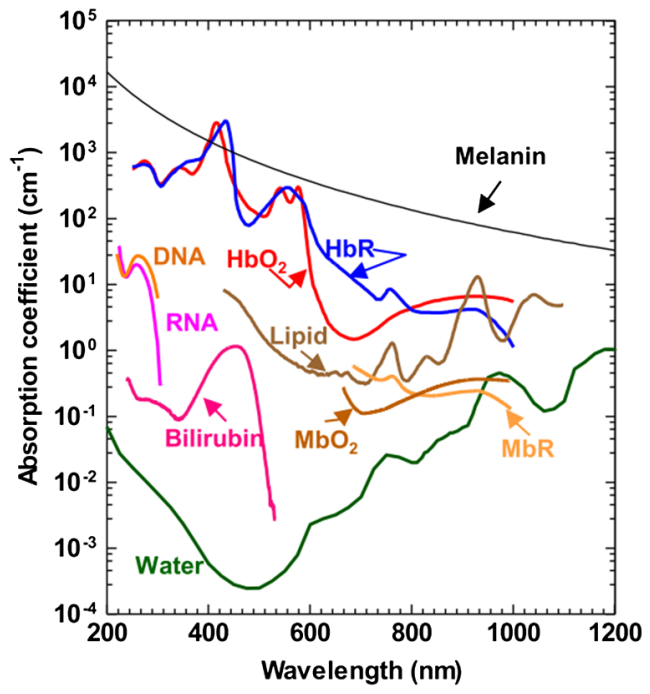

Fig. 6 PAT imaging of endogenous contrast agents: DNA/RNA (a), hemoglobin (b), and melanin (c). Reproduced with permission from Ref. 14.

$$
\begin{aligned}
p\left(\lambda_{1}\right) & =k \mu_{a}\left(\lambda_{1}\right) F\left(\lambda_{1}\right) \\
& =k\left[\varepsilon_{\mathrm{HbR}}\left(\lambda_{1}\right) C_{\mathrm{HbR}}+\varepsilon_{\mathrm{HbO}_{2}}\left(\lambda_{1}\right) C_{\mathrm{HbO}_{2}}\right] F\left(\lambda_{1}\right),
\end{aligned}
$$

where $p\left(\lambda_{1}\right)$ is the measured PA amplitude at wavelength $\lambda_{1}, k$ is a system constant, $\mu_{a}$ is the blood absorption coefficient, $F$ is the optical fluence. $\varepsilon_{\mathrm{HbR}}$ and $\varepsilon_{\mathrm{HbO}_{2}}$ are the molar extinction coefficients of $\mathrm{HbR}$ and $\mathrm{HbO}_{2}$, respectively, and $C_{\mathrm{HbR}}$ and $C_{\mathrm{HbO}_{2}}$ are the concentrations of $\mathrm{HbR}$ and $\mathrm{HbO}_{2}$, respectively. For a given system, $k$ is a constant. In OR-PAM, which works within the optical ballistic regime, $F$ can be corrected for by measuring the surface optical fluence. Thus, by performing measurements at two wavelengths, the relative concentrations of $\mathrm{HbR}$ and $\mathrm{HbO}_{2}$ can be quantified

$$
C_{\mathrm{HbR}}=k \frac{p\left(\lambda_{1}\right) \varepsilon_{\mathrm{HbR}}\left(\lambda_{2}\right)-p\left(\lambda_{2}\right) \varepsilon_{\mathrm{HbR}}\left(\lambda_{1}\right)}{\varepsilon_{\mathrm{HbR}}\left(\lambda_{1}\right) \varepsilon_{\mathrm{HbO}_{2}}\left(\lambda_{2}\right)-\varepsilon_{\mathrm{HbR}}\left(\lambda_{2}\right) \varepsilon_{\mathrm{HbO}_{2}}\left(\lambda_{1}\right)},
$$

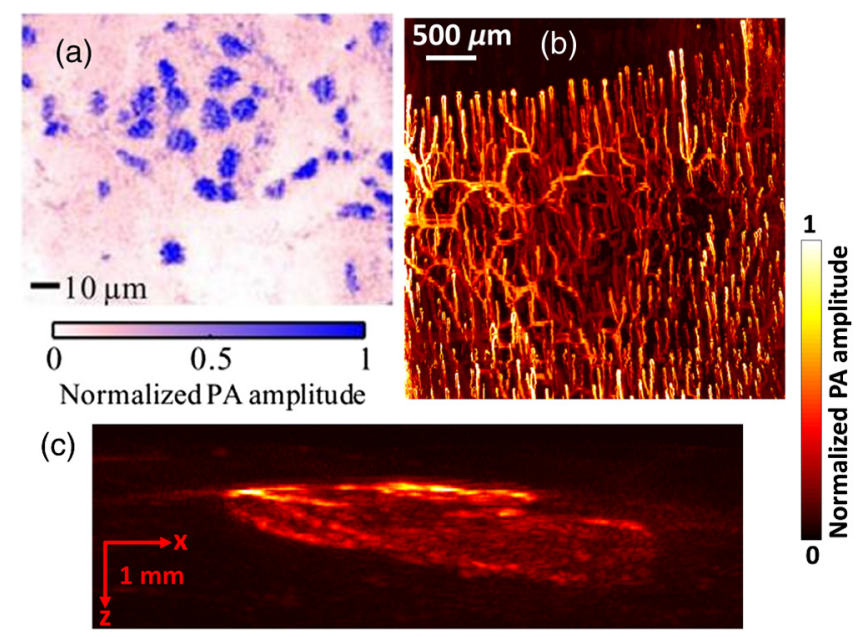

Fig. 7 Absorption spectra of the main endogenous pigments in tissue at normal concentrations. $\mathrm{HbO}_{2}$, oxygenated hemoglobin; $\mathrm{HbR}$, deoxygenated hemoglobin; $\mathrm{MbO}_{2}$, oxygenated myoglobin; and $\mathrm{MbR}$, deoxygenated myoglobin. Reproduced with permission from Refs. 79, 19, and 54. 
and

$$
C_{\mathrm{HbO}_{2}}=k \frac{p\left(\lambda_{1}\right) \varepsilon_{\mathrm{HbR}}\left(\lambda_{2}\right)-p\left(\lambda_{2}\right) \varepsilon_{\mathrm{HbR}}\left(\lambda_{1}\right)}{\varepsilon_{\mathrm{HbR}}\left(\lambda_{2}\right) \varepsilon_{\mathrm{HbO}_{2}}\left(\lambda_{1}\right)-\varepsilon_{\mathrm{HbR}}\left(\lambda_{1}\right) \varepsilon_{\mathrm{HbO}_{2}}\left(\lambda_{2}\right)},
$$

where $\lambda_{2}$ stands for the second wavelength. By definition, the $\mathrm{sO}_{2}$ value can be calculated as

$$
\begin{aligned}
& \mathrm{sO}_{2}=\frac{C_{\mathrm{HbO}_{2}}}{C_{\mathrm{HbO}_{2}}+C_{\mathrm{HbR}}} \\
& =\frac{p\left(\lambda_{1}\right) \varepsilon_{\mathrm{HbR}}\left(\lambda_{2}\right)-p\left(\lambda_{2}\right) \varepsilon_{\mathrm{HbR}}\left(\lambda_{1}\right)}{p\left(\lambda_{1}\right)\left[\varepsilon_{\mathrm{HbR}}\left(\lambda_{2}\right)-\varepsilon_{\mathrm{HbO}_{2}}\left(\lambda_{2}\right)\right]-p\left(\lambda_{2}\right)\left[\varepsilon_{\mathrm{HbR}}\left(\lambda_{1}\right)-\varepsilon_{\mathrm{HbO}_{2}}\left(\lambda_{1}\right)\right]} .
\end{aligned}
$$

However, in AR-PAM and PACT, which work in the optical (quasi)diffusive regime, it is challenging to correct for $F$. So far, several methods have been proposed to address this issue and achieve more accurate $\mathrm{sO}_{2}$ measurement in the optical (quasi)diffusive regime, ${ }^{100-103}$ such as directly fitting the temporal profiles of PA signals, ${ }^{100,103}$ analyzing their acoustic spectra, ${ }^{101}$ and measuring their dynamics. ${ }^{102}$

\subsection{Exogenous Contrast Agents}

Compared with endogenous contrast agents, exogenous ones can be engineered to absorb at specific wavelengths to maximize their detection sensitivities. In addition, exogenous agents can be made to bind to only certain molecules; thus, these molecules can be selectively imaged by PAT. So far, a variety of exogenous agents have been developed,,${ }^{9,104,105}$ including nanoparticles, ${ }^{106-109}$ organic dyes, ${ }^{10-115}$ and proteins. ${ }^{116,117}$ Recently, for PA imaging, agents with specially designed functions have been developed, such as photosensitizing, ${ }^{118,119}$ activatable, ${ }^{120}$ and switchable agents. ${ }^{121-123}$ Note that since hemoglobin and water have strong absorption in the visible and midinfrared regions, respectively, most of these exogenous agents are designed to work in the near-infrared window, i.e., from 700 to $1350 \mathrm{~nm}$. Usually, to enable more accurate spectral decomposition, two or more wavelengths are chosen to image blood and exogenous contrasts separately. Figure 8 shows two examples of using exogenous contrast agents in PA imaging: methylene blue ${ }^{115}$ and gold nanoparticles. ${ }^{108}$ Before the contrasts were injected, only blood vessels could be detected, as shown in Figs. 8(a) and 8(c). However, after injecting the contrasts, a sentinel lymph node and tumor appeared with high contrast, as seen in Figs. 8(b) and 8(d), respectively.

\section{Recent Applications}

With its multiparameter and multiscale imaging capability, PAT has been applied in many different disciplines, including cardiology, ${ }^{77,124,125}$ dermatology, ${ }^{126-129}$ oncology, ${ }^{130-133}$ ophthalmology, ${ }^{67,69,134-136}$ gastroenterology, ${ }^{36-38,40,137-140}$ hematology, ${ }^{141-143}$ and neurology. ${ }^{144-147}$ In terms of imaging locations, PAT can be used for human breast imaging ${ }^{148-150}$ and smallanimal imaging of the brain, ${ }^{70,151,152}$ ear, ${ }^{153-155}$ eye, ${ }^{134-136}$ liver, ${ }^{15}$ intestine, ${ }^{156}$ and skin. ${ }^{54,90}$ In terms of functionality, PAT has been widely used for anatomical, ${ }^{157,158}$ functional, ${ }^{159-161}$ molecular, ${ }^{9,82}$ and metabolic imaging. ${ }^{162,163}$ As for object size, PAT can detect objects ranging from organelles to human organs or whole-body small animals. ${ }^{11}$ PAT has been used for both small animal imaging (such as zebrafish, ${ }^{164}$ mice, ${ }^{165}$ rats, ${ }^{151}$
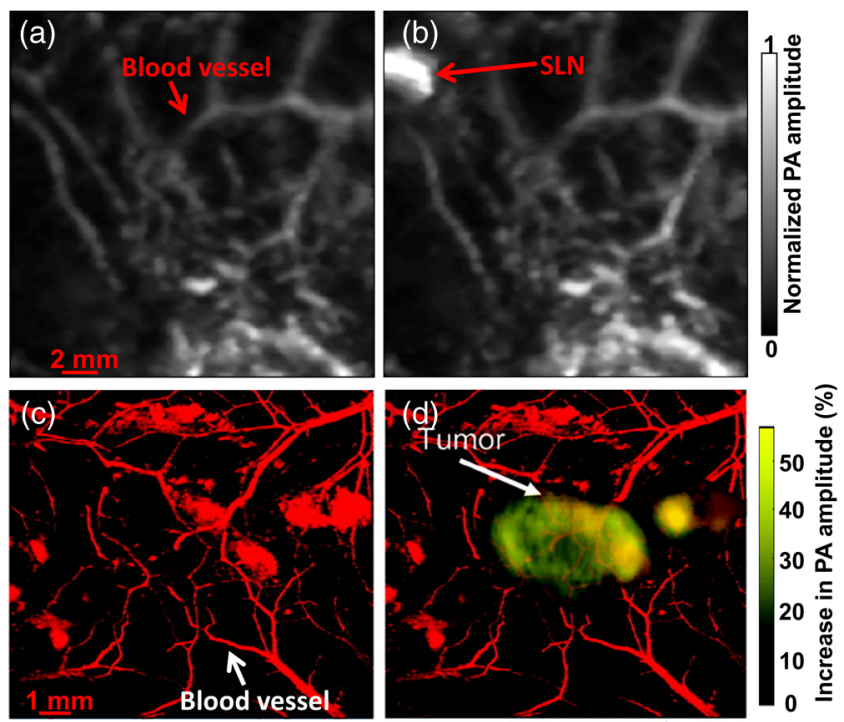

Fig. 8 PAT imaging aided by exogenous contrast agents. PA images acquired before (a) and $~ 52$ min after (b) methylene blue injection, showing a dramatic PA signal increase in a sentinel lymph node (SLN). PA images acquired (c) before and (d) $6 \mathrm{~h}$ after gold nanoparticle injection, showing a significant $P A$ signal increase in a melanoma tumor. Reproduced with permission from Refs. 115 (a) and (b) and 108 (c) and (d).

and rabbits ${ }^{166}$ ) and human imaging. ${ }^{72}$ In this review paper, rather than cover details of all the applications, we will simply list the most recent advances and significant applications of PAT.

\subsection{High Speed Imaging of Mouse Brain Functions}

PAT has been extensively applied for brain studies. Wang et al. ${ }^{47}$ reported the first in vivo mouse brain function study with PAT. Using a circular-view PACT system, they imaged rat brain responses to vibrational stimulations to whiskers. Because a single-element ultrasonic transducer with a center frequency of $3.5 \mathrm{MHz}$ was used, both the frame rate and spatial resolution were limited. With newer techniques, both the frame rate and spatial resolution have been significantly improved. For example, using an ultrasonic transducer array with 512 elements, mouse brain imaging at one frame per $1.25 \mathrm{~s}$ has been achieved. ${ }^{48}$ Using OR-PAM, optical resolution mouse brain images, i.e., at micrometer or submicrometer level resolution, were obtained. ${ }^{167}$

Recently, Yao et al. ${ }^{70}$ reported fast functional PAM (ffPAM) for mouse brain function imaging in action. Working as ORPAM, this system has a lateral resolution of $\sim 3 \mu \mathrm{m}$ and an axial resolution of $\sim 15 \mu \mathrm{m}$. Using a water-immersed microelectromechanical system (MEMS) scanning mirror along with a $500 \mathrm{kHz}$ repetition rate laser, ffPAM has a 2-D frame rate of $400 \mathrm{~Hz}$ over a $3-\mathrm{mm}$ scanning range. With a $3 \times 2 \mathrm{~mm}^{2}$ field of view, a 3-D volumetric rate of $1 \mathrm{~Hz}$ can be achieved. By using a single-wavelength pulse-width-based method $\left(\mathrm{PW}-\mathrm{sO}_{2}\right)$, ffPAM can perform high speed imaging of $\mathrm{sO}_{2}$ up to a 1-D rate of $100 \mathrm{kHz}$. As shown in Fig. 9(a), to implement the $\mathrm{PW}_{-} \mathrm{sO}_{2}$ method, lasers with different pulse widths are used, i.e., 3 ps and 3 ns. Figure 9(b) shows a representative PA image of the mouse brain with the skull intact, where the cortex vasculature can be visualized in detail. With the $\mathrm{PW}-\mathrm{sO}_{2}$ method, only $40 \mathrm{~s}$ were required to acquire the $\mathrm{sO}_{2}$ map shown in Fig. 9(c). 
(a)

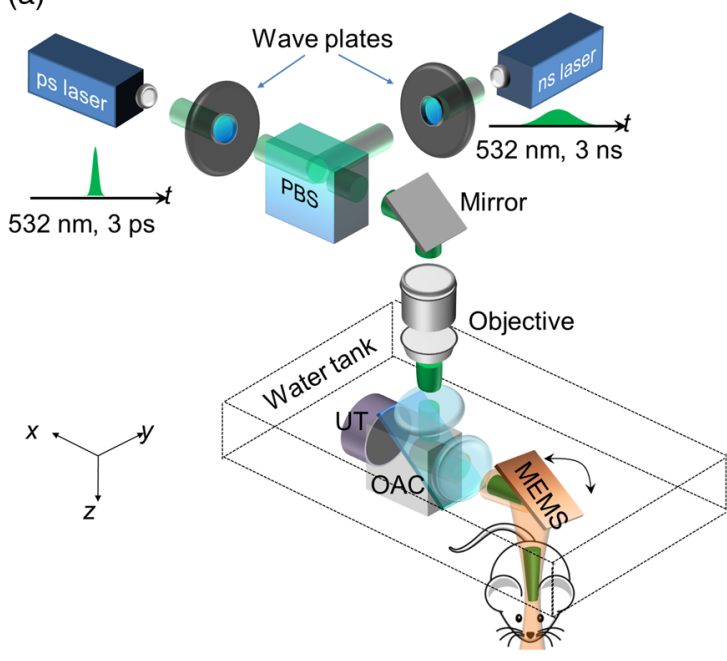

(b)

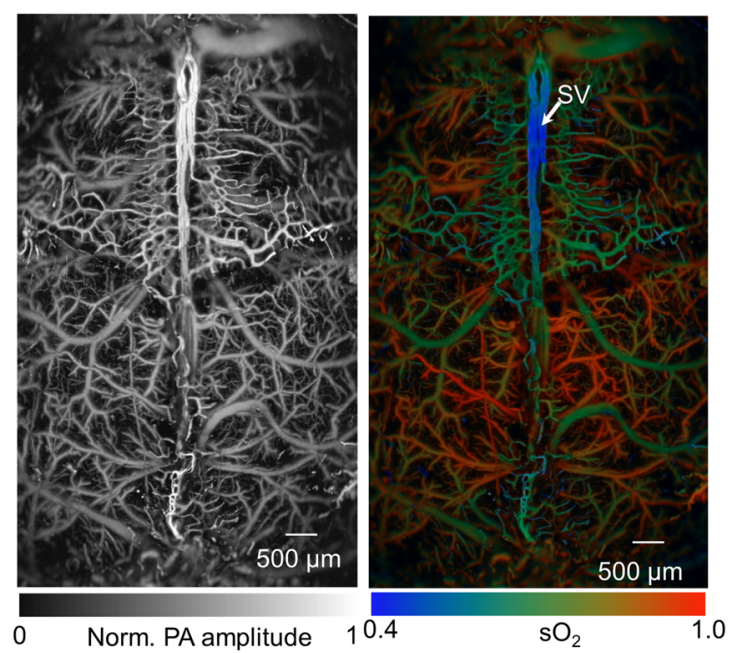

Fig. 9 Fast functional PAM (ffPAM) of the mouse brain. (a) Schematic of the ffPAM system. MEMS, microelectromechanical system; OAC, optical-acoustic combiner; PBS, polarizing beam splitter; and UT, ultrasonic transducer. Anatomical (b) and (c) functional images of the mouse brain. $\mathrm{sO}_{2}$, oxygen saturation of hemoglobin; and SV, skull vessel. Reproduced with permission from Ref. 70.

To further demonstrate the fast functional imaging capability, the authors studied mouse cortical responses to electrical stimulations of the hindlimbs. As shown in Fig. 10(a), upon stimulations, PA signals in the corresponding regions increased. In addition, $\mathrm{sO}_{2}$ levels increased in veins and deep capillary beds upon stimulation, as shown in Fig. 10(b). However, there was no arterial $\mathrm{sO}_{2}$ response, which is consistent with the fact that arterial blood had not reached the capillaries for oxygen exchange and thus maintained a high oxygenation level.

\subsection{Sentinel Lymph Node Biopsy Guidance in Patients with Breast Cancer}

Sentinel lymph node (SLN) biopsy is a standard of care in diagnosing cancer, including breast and melanoma cancers. Because (a)
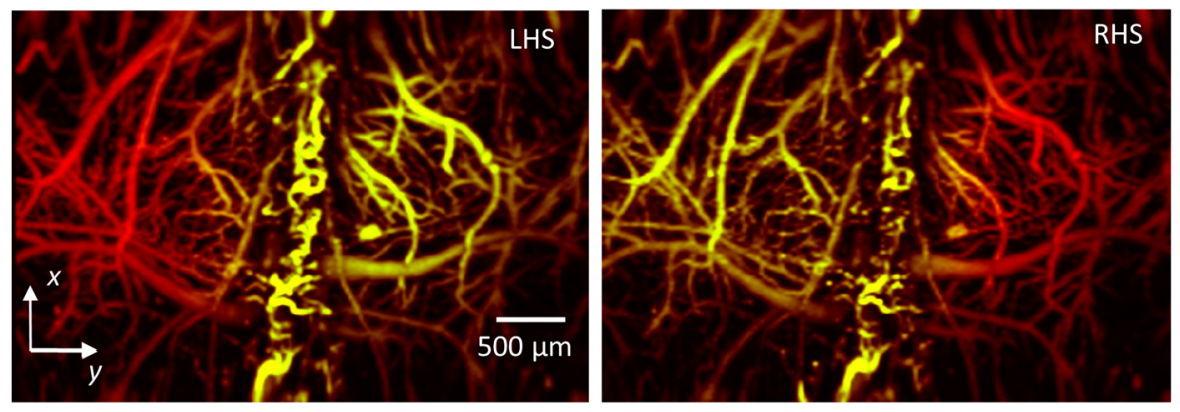

Fractional change (\%)

$$
0
$$

20

(b)
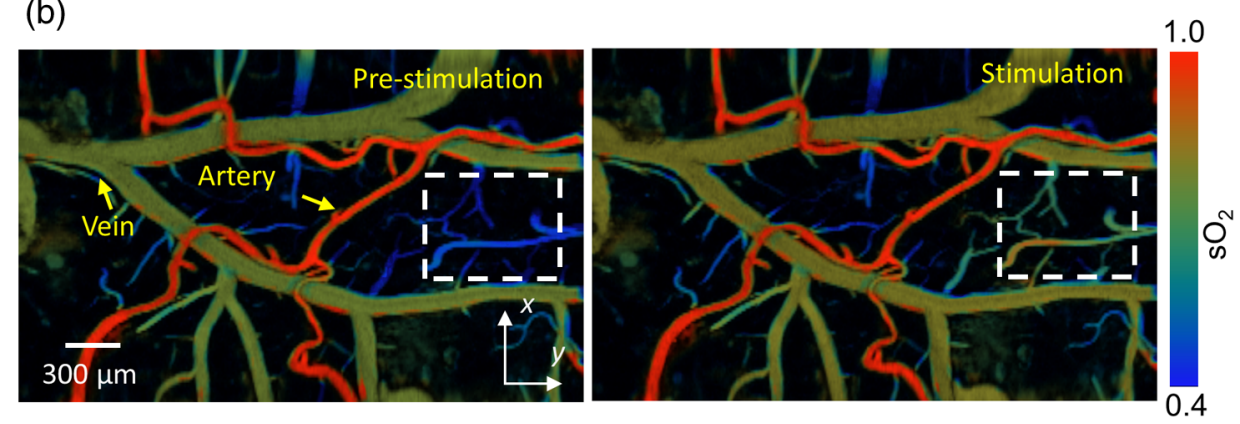

Fig. 10 ffPAM of brain responses to electrical stimulations of the hindlimbs of mice. (a) Fractional PA amplitude changes during left hindlimb stimulation (LHS) and right hindlimb stimulation (RHS). (b) $\mathrm{sO}_{2}$ imaging (marked by the dashed box) before and during stimulations of the left hindlimb. Reproduced with permission from Ref. 70. 


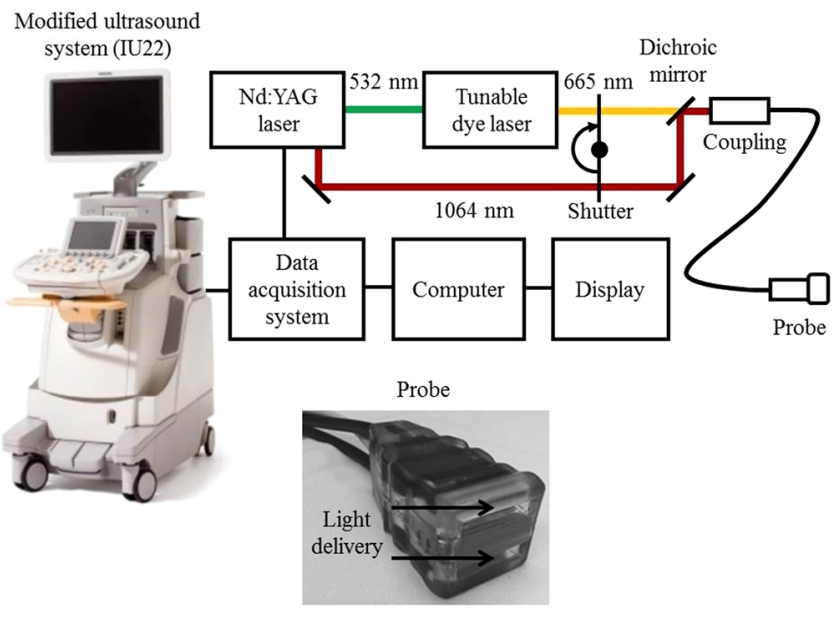

Fig. 11 Schematic of the dual-modality ultrasound and photoacoustic system for SLN detection. Reproduced with permission from Ref. 168.

the SLN is the first node in the lymphatic system that drains a tumor site, metastasis can be diagnosed by SLN biopsy. A positive biopsy result suggests that cancer has spread to the node and probably to distant organs. Recently, a handheld dual-modality ultrasound (US) and PAT system was used to accurately identify an SLN and thus to precisely guide SLN biopsy. ${ }^{168}$

The dual-modality system was modified from a clinical US scanner (iU22, Philips Healthcare). To highlight SLNs in patients, a very common clinical contrast agent, methylene blue, was used. As shown in Fig. 11, laser pulses at two wavelengths of 665 and $1064 \mathrm{~nm}$ spectrally differentiated methylene blue from other major absorbers, such as blood. The pulse width was around $6.5 \mathrm{~ns}$, and the pulse repetition rate was $10 \mathrm{~Hz}$. With a custom-built data acquisition computer, this system could achieve a frame rate of $5 \mathrm{~Hz}$ for coregistered US and PA imaging. To improve the detection sensitivity and operation convenience, the light delivery fiber bundles and ultrasonic transducer array were integrated into a single probe, as shown in Fig. 11. Because the probe is handheld, it is convenient for physicians to operate for SLN biopsy guidance.

As shown in Fig. 12, by combining both US and PA, the SLN in a patient with breast cancer can be clearly detected. In addition, taking advantage of its high frame rate, this system can provide guidance for fine needle aspiration biopsy (FNAB) with minimal invasiveness. Because both the SLN and the needle can be imaged with high contrast, FNAB can be performed with high accuracy.

\subsection{Multiscale Photoacoustic Tomography with Photo-Switchable Protein Contrast}

A reversibly switchable bacterial phytochrome, Rhodopseudomonas palustris (BphP1), has been recently combined with PAT for deep molecular imaging with improved detection sensitivity and spatial resolution. ${ }^{121} \mathrm{BphP1}$ has two states: Pfr and Pr. Upon 730- to 790-nm light illumination, it undergoes a $\mathrm{Pfr} \rightarrow \operatorname{Pr}$ photoconversion; while upon 630- to 690-nm light illumination, $\mathrm{Pr} \rightarrow \mathrm{Pfr}$ photoconversion happens. For simplicity, the Pfr state of BphP1 was denoted as the ON state and the Pr state as the OFF state. In the reported work, $780 \mathrm{~nm}$ light was used for $\mathrm{Pfr} \rightarrow \mathrm{Pr}$ photoconversion, and 630-nm light was used for $\mathrm{Pr} \rightarrow \mathrm{Pfr}$ photoconversion. Because the background absorbers, primarily blood, did not have the same switchable properties as BphP1, taking differential images largely suppressed the background signals and thus increased the detection sensitivity for BphP1-expressing tumors in deep tissue.

First implementing BphP1 with a circular-view PACT system, the authors observed a noise-equivalent detection sensitivity of 20 U87 human glioblastoma cells expressing BphP1, as shown in Fig. 13(a). With the single-wavelength differential method, the CNR was about 34-fold higher than the two-wavelength spectral unmixing method. In the in vivo experiment, a mouse was imaged 1 week after injection of BphP1-expressing U87 cells into its left kidney. As shown in Fig. 13(b), although major organs, such as the skin, kidneys, spleen, and bladder, can all be clearly imaged, the tumor in the left kidney could not be detected because of the overwhelming blood signals. However, after 20 cycles of photoswitching, the differential image showed the tumor at depths up to $8 \mathrm{~mm}$ with high contrast, as seen in Figs. 13(b) and 13(c). The line profiles in Fig. 13(b) show that while the background blood signals remained unchanged, the photoswitchable tumor had clearly different signals in the ON- and OFF-state images [Fig. 13(d)]. A histological examination taken after PA imaging confirmed the tumor, as shown in Fig. 13(e). Another tumor detection experiment, in a mouse brain, also showed the superior sensitivity of the BphP1enhanced PACT system, as shown in Fig. 13(f).

Combined with a high NA (1.4 in this work) OR-PAM system [Fig. 14(a)], this reversely switchable protein can also be used for super-resolution PA imaging (RS-SPAM). As shown in Fig. 14(b), because the switching-off rate is proportional to the local excitation intensity, PA signals from the center of the excitation spot will decay faster than those from the periphery. By fitting the nonlinear signal-decay process, a high-order coefficient can be extracted and thus subdiffraction resolution

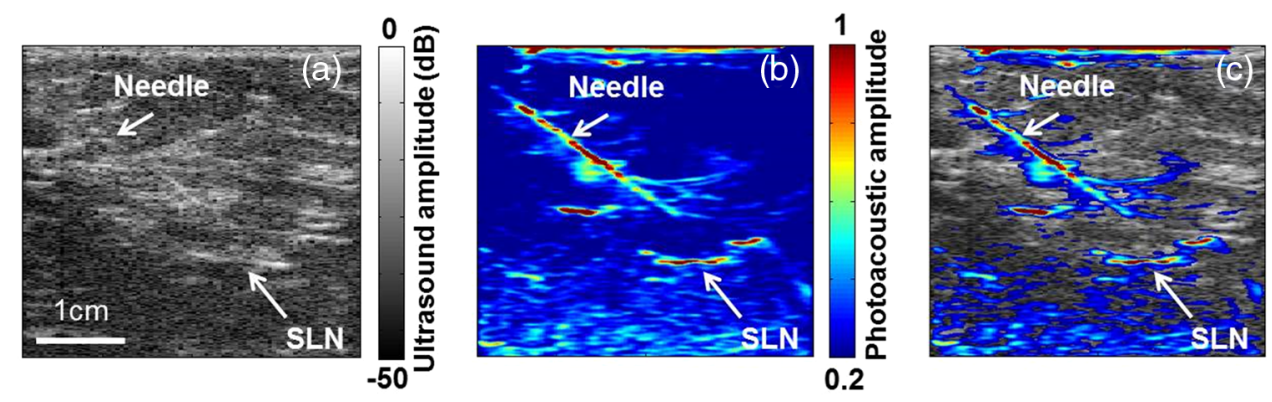

Fig. 12 In vivo images of a human axilla acquired by US (a), PA (b), and both (c). Reproduced with permission from Ref. 168. 
(a)

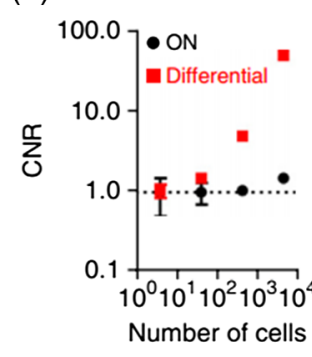

(c)

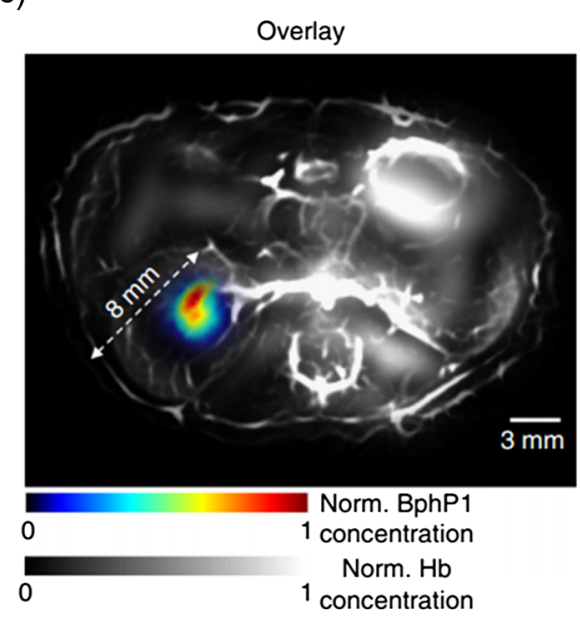

(b)

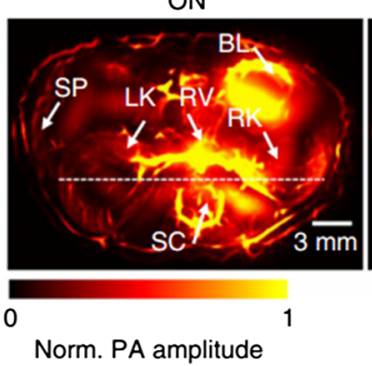

(d)

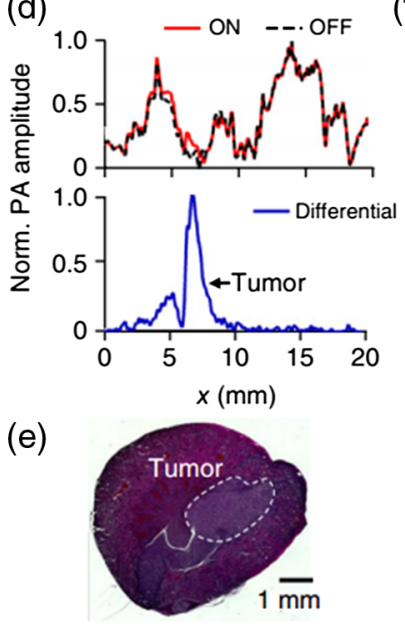

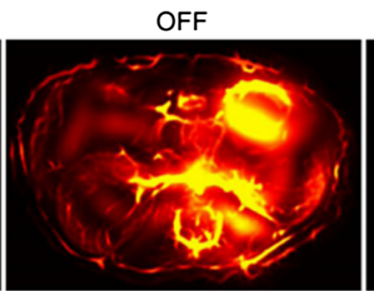

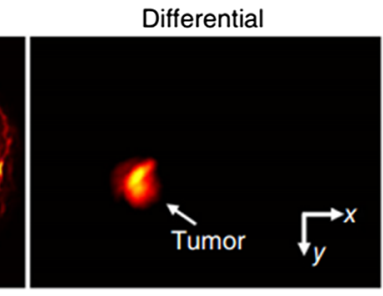

(f)

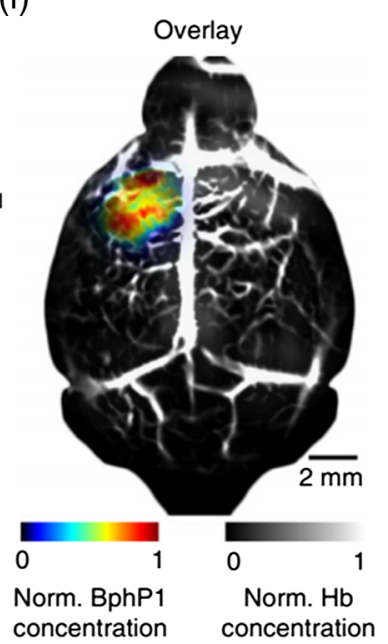

Fig. 13 Circular-view PACT system combined with reversely switchable BphP1 for deep imaging. (a) Contrast-to-noise ratio (CNR) of U87 cells imaged by PACT at 10-mm depth. (b) Whole-body mouse images acquired with BphP1 at different state. The differential image clearly shows that the tumor is at the left kidney. (c) An overlay of the differential image (in color) and the blood-dominated OFF-state image (in grayscale). (d) Normalized PA amplitude along the dashed line in (b), showing the contrast enhancement of the tumor in the differential image. (e) A histology image of the left kidney showing the tumor region. (f) PACT image of a mouse brain with a U87 tumor expressing BphP1. The tumor was $\sim 3 \mathrm{~mm}$ beneath the scalp. Reproduced with permission from Ref. 121.

can be achieved. As shown in Figs. 14(c)-14(g), RS-SPAM showed much finer lateral and axial resolutions. The lateral resolution was quantified to be $\sim 141 \mathrm{~nm}$, which is about twofold better than that of conventional PAM, and the axial resolution was around $400 \mathrm{~nm}$ in RS-SPAM, which was around 75 times better than that of conventional PAM.

\section{Summary}

In summary, PAT is a highly scalable imaging modality with major implementations of PAM and PACT. In PAM, light is focused into the target and a focused transducer is typically used for the signal detection. Thus, its lateral resolution is determined by either the optical focus (OR-PAM) or acoustic focus (AR-PAM), depending on which one is tighter. In OR-PAM, the lateral resolution is given by $0.51 \lambda_{\mathrm{o}} / \mathrm{NA}_{\mathrm{o}}$, where $\lambda_{\mathrm{o}}$ is the light wavelength, and $\mathrm{NA}_{\mathrm{o}}$ is the NA of the optical focusing lens. In AR-PAM, the lateral resolution is given by $0.72 \lambda_{\mathrm{a}} / \mathrm{NA}_{\mathrm{a}}$, where $\lambda_{\mathrm{a}}$ is the central acoustic wavelength of the ultrasonic transducer, and $\mathrm{NA}_{\mathrm{a}}$ is the NA of the acoustic lens. The difference in the scaling factors arises because optical excitation is based on light intensity, while ultrasonic detection is based on acoustic amplitude. In both cases, the axial resolution is given by $0.88 c / \Delta f$, where $c$ is the speed of sound in soft tissue, and $\Delta f$ is the bandwidth of the ultrasonic transducer. This axial resolution formula also applies to PACT systems. However, unlike PAM, lateral resolutions in PACT are usually not a constant. Thus, we can see that by choosing different optical focusing lenses or ultrasonic transducers, both lateral and axial resolution can be changed. Super optical resolution has been achieved in ORPAM. ${ }^{169}$ In addition, because acoustic attenuation in tissues increases with the acoustic frequency, ultrasonic transducers with different center frequencies should be chosen according to the desired imaging penetration limit. The maximum penetration achieved in PA images is $\sim 7 \mathrm{~cm}$ with a lateral resolution about $720 \mu \mathrm{m} .^{11}$

PAT is also a multiparameter imaging modality. In most of the cases, because hemoglobin provides the highest contrast in biological imaging, most studies focus on extracting and studying blood-related parameters, such as blood vessel diameter, ${ }^{31}$ blood flow speed, ${ }^{170,171}$ hemoglobin oxygen concentration, ${ }^{19,31}$ blood pulse wave velocity, ${ }^{172}$ and the metabolic rate of oxygen. ${ }^{162}$ PAT has provided valuable information for studying vasculature-related diseases, such as stroke, ${ }^{173}$ diabetes, ${ }^{174}$ and atherosclerosis. ${ }^{98,175,176}$ In addition, because neural activities are closely related to hemodynamics, measuring these hemodynamic parameters is also useful for neurological studies, such as studies on epilepsy, ${ }^{177}$ resting state functional connectivity, ${ }^{152}$ and stimulation responses. ${ }^{147}$ Because of its high contrast in comparison to other absorbers, such as melanin ${ }^{54}$ and DNA/RNA, ${ }^{79}$ PAT can also image other important biological 
(a)

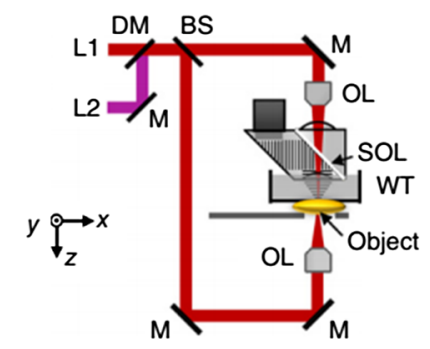

(d)

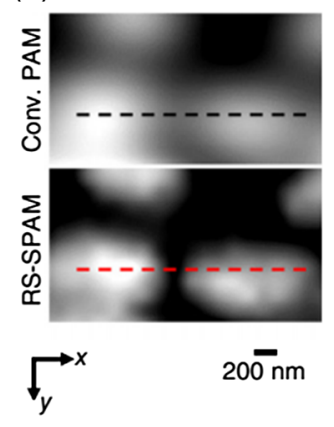

(b)

(e)

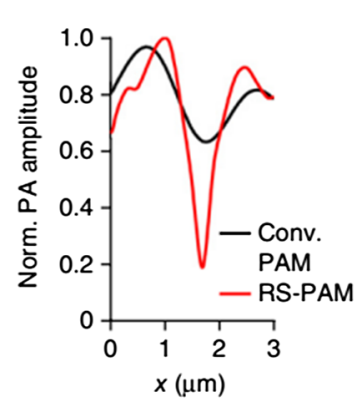

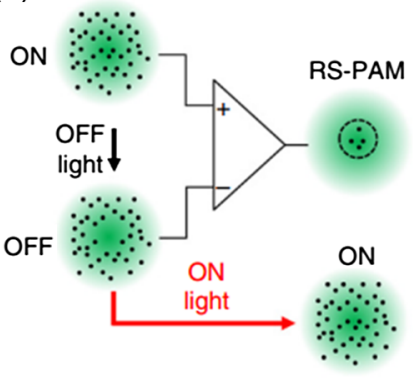

(f) (c)

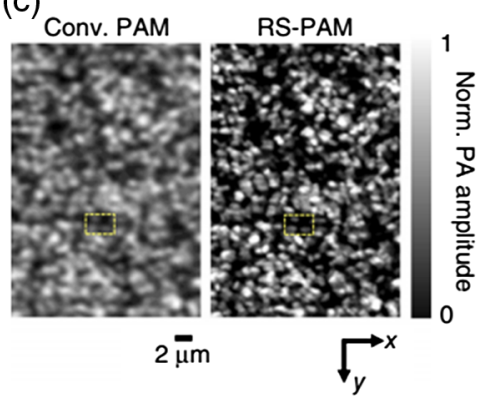

(g)
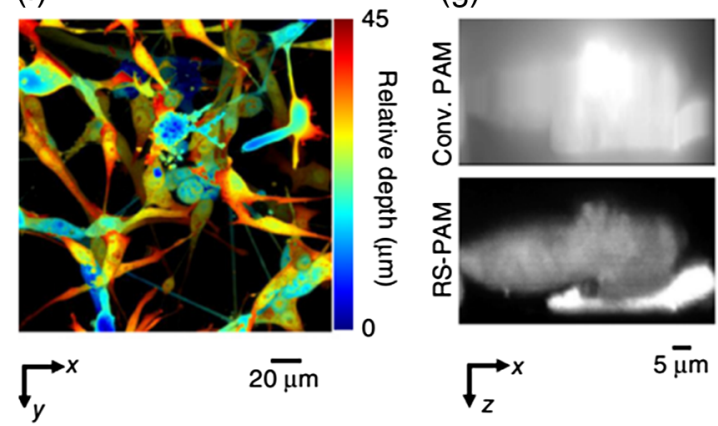

Fig. 14 Reversely switchable BphP1-enhanced super-resolution PAM (RS-SPAM). (a) Schematic of the RS-SPAM system. (b) Subdiffraction-resolution principle. In the diffraction-limited excitation volume (green), part of the ON-state BphP1 molecules (black dots) are switched to the OFF state, where the switching rate is proportional to the local optical intensity. The differential signals generate super-resolution images. (c) Conventional (Conv.) PAM and RS-SPAM images of BphP1-expressing bacteria, showing the superior lateral resolution of RS-SPAM. (d) Zoomed-in images of the dashed box areas in (c). (e) Normalized PA amplitude along the dashed line in (d). (f) Depth-encoded RS-SPAM image of BphP1-expressing U87 cells. (g) $x-z$ cross images of two stacked U87 cells, showing the finer axial resolution of RS-SPAM. Reproduced with permission from Ref. 121.

parameters. Imaging melanin can provide the depth of melanoma, as well as its rate of growth and metastatic rate, ${ }^{54}$ which are all very important parameters in diagnosing and treating melanoma patients. Imaging DNA/RNA provides a tool for label-free measurement of cell nuclear density, which potentially can be used for tumor demarcation.

PAT has become one of the fastest growing fields in biomedical imaging. So far, PAT has translated several important applications into clinics, which may help solve existing problems in health care. For example, noninvasive detection of SLNs ${ }^{168}$ in patients can potentially provide minimally invasive cancer staging, and quantification of melanoma depth ${ }^{54}$ can potentially guide more accurate surgeries, both reducing morbidity and costs. With advances in new techniques, we anticipate that PAT will provide valuable information for disease diagnosis as well as treatment.

\section{Acknowledgments}

The authors would like to thank Professor James Ballard for manuscript editing. This work was sponsored in part by National Institutes of Health Grant Nos. DP1 EB016986 (NIH Director's Pioneer Award), R01 CA186567 (NIH Director's Transformative Research Award), and S10 RR026922. L. V. W. has a financial interest in Microphotoacoustics, Inc., which, however, did not support this work.

\section{References}

1. L. V. Wang and L. Gao, "Photoacoustic microscopy and computed tomography: from bench to bedside," Annu. Rev. Biomed. Eng. 16, 155-185 (2014).
2. L. V. Wang, "Tutorial on photoacoustic microscopy and computed tomography," IEEE J. Sel. Top. Quantum Electron. 14(1), 171-179 (2008).

3. P. Beard, "Biomedical photoacoustic imaging," Interface Focus 1(4), 602-631 (2011).

4. J. Yao and L. V. Wang, "Photoacoustic tomography: fundamentals, advances and prospects," Contrast Media Mol. Imaging 6(5), 332-345 (2011).

5. V. Ntziachristos, "Going deeper than microscopy: the optical imaging frontier in biology," Nat. Methods 7(8), 603-614 (2010).

6. S. Hu and L. V. Wang, "Photoacoustic imaging and characterization of the microvasculature," J. Biomed. Opt. 15(1), 011101 (2010).

7. C. Li and L. V. Wang, "Photoacoustic tomography and sensing in biomedicine," Phys. Med. Biol. 54(19), R59-R97 (2009).

8. L. V. Wang, "Multiscale photoacoustic microscopy and computed tomography," Nat. Photonics 3(9), 503-509 (2009).

9. C. Kim, C. Favazza, and L. V. Wang, "In vivo photoacoustic tomography of chemicals: high-resolution functional and molecular optical imaging at new depths," Chem. Rev. 110(5), 2756-2782 (2010).

10. X. Cai et al., "Photoacoustic microscopy in tissue engineering," Mater. Today 16(3), 67-77 (2013).

11. L. V. Wang and S. Hu, "Photoacoustic tomography: in vivo imaging from organelles to organs," Science 335(6075), 1458-1462 (2012).

12. J. Yao and L. V. Wang, "Photoacoustic microscopy," Laser Photon. Rev. 7(5), 758-778 (2013).

13. J. Yao, L. Song, and L. V. Wang, "Photoacoustic microscopy superdepth, superresolution, and superb contrast," IEEE Pulse 6(3), 34-37 (2015).

14. J. Yao and L. V. Wang, "Sensitivity of photoacoustic microscopy," Photoacoustics 2(2), 87-101 (2014).

15. J. Xia and L. V. Wang, "Small-animal whole-body photoacoustic tomography: a review," IEEE Trans. Bio-Med. Eng. 61(5), 1380-1389 (2014).

16. J. Yao and L. V. Wang, "Photoacoustic brain imaging: from microscopic to macroscopic scales," Neurophotonics 1(1), 011003 (2014). 
17. S. Mallidi, G. P. Luke, and S. Emelianov, "Photoacoustic imaging in cancer detection, diagnosis, and treatment guidance," Trends Biotechnol. 29(5), 213-221 (2011).

18. B. Cox et al., "Quantitative spectroscopic photoacoustic imaging: a review," J. Biomed. Opt. 17(6), 061202 (2012).

19. S. Hu and L. V. Wang, "Optical-resolution photoacoustic microscopy: auscultation of biological systems at the cellular level," Biophys. J. 105(4), 841-847 (2013).

20. L. V. Wang, "Prospects of photoacoustic tomography," Med. Phys. 35(12), 5758-5767 (2008).

21. L. V. Wang, "Multiscale photoacoustic microscopy and computed tomography," Nat. Photonics 3(9), 503-509 (2009).

22. D. K. Yao et al., "Photoacoustic measurement of the Gruneisen parameter of tissue," J. Biomed. Opt. 19(1), 017007 (2014).

23. L. Gao et al., "Intracellular temperature mapping with fluorescenceassisted photoacoustic-thermometry," Appl. Phys. Lett. 102(19) (2013).

24. M. H. Xu and L. H. V. Wang, "Photoacoustic imaging in biomedicine," Rev. Sci. Instrum. 77(4), 041101 (2006).

25. M. H. Xu and L. H. V. Wang, "Universal back-projection algorithm for photoacoustic computed tomography," Phys. Rev. E 71(1), 016706 (2005).

26. B. E. Treeby and B. T. Cox, "k-Wave: MATLAB toolbox for the simulation and reconstruction of photoacoustic wave fields," J. Biomed. Opt. 15(2), 021314 (2010).

27. M. Xu and L. V. Wang, "Analytic explanation of spatial resolution related to bandwidth and detector aperture size in thermoacoustic or photoacoustic reconstruction," Phys. Rev. E 67(5 Pt 2), 056605 (2003).

28. K. Wang et al., "Investigation of iterative image reconstruction in three-dimensional optoacoustic tomography," Phys. Med. Biol. 57(17), 5399-5423 (2012).

29. K. Wang et al., "Discrete imaging models for three-dimensional optoacoustic tomography using radially symmetric expansion functions," IEEE Trans. Med. Imaging 33(5), 1180-1193 (2014).

30. K. Wang et al., "An imaging model incorporating ultrasonic transducer properties for three-dimensional optoacoustic tomography," IEEE Trans. Med. Imaging 30(2), 203-214 (2011).

31. S. Hu, K. Maslov, and L. V. Wang, "Second-generation optical-resolution photoacoustic microscopy with improved sensitivity and speed," Opt. Lett. 36(7), 1134-1136 (2011).

32. K. Maslov et al., "Optical-resolution photoacoustic microscopy for in vivo imaging of single capillaries," Opt. Lett. 33(9), 929-931 (2008).

33. H. F. Zhang, K. Maslov, and L. H. V. Wang, "In vivo imaging of subcutaneous structures using functional photoacoustic microscopy," Nat. Protoc. 2(4), 797-804 (2007).

34. H. F. Zhang et al., "Functional photoacoustic microscopy for highresolution and noninvasive in vivo imaging," Nat. Biotechnol. 24(7), 848-851 (2006).

35. K. Maslov, G. Stoica, and L. H. V. Wang, "In vivo dark-field reflectionmode photoacoustic microscopy," Opt. Lett. 30(6), 625-627 (2005).

36. J. M. Yang et al., "Photoacoustic endoscopy," Opt. Lett. 34(10), 15911593 (2009).

37. J. M. Yang et al., "Catheter-based photoacoustic endoscope," J. Biomed. Opt. 19(6), 066001 (2014).

38. J. M. Yang et al., "Optical-resolution photoacoustic endomicroscopy in vivo," Biomed. Opt. Express. 6(3), 918-932 (2015).

39. J. M. Yang et al., "Simultaneous functional photoacoustic and ultrasonic endoscopy of internal organs in vivo," Nat. Med. 18(8), 1297-1302 (2012).

40. C. Y. Li et al., "Urogenital photoacoustic endoscope," Opt. Lett. 39(6), 1473-1476 (2014).

41. C. Yeh et al., "Three-dimensional arbitrary trajectory scanning photoacoustic microscopy," J. Biophotonics 8(4), 303-308 (2015).

42. J. Y. Liang et al., "Random-access optical-resolution photoacoustic microscopy using a digital micromirror device," Opt. Lett. 38(15), 2683-2686 (2013).

43. R. A. Kruger et al., "Thermoacoustic molecular imaging of small animals," Mol. Imaging 2(2), 113-123 (2003).

44. H. P. Brecht et al., "Whole-body three-dimensional optoacoustic tomography system for small animals," J. Biomed. Opt. 14(6), 064007 (2009).

45. R. A. Kruger et al., "Photoacoustic angiography of the breast," Med. Phys. 37(11), 6096-6100 (2010).

46. R. A. Kruger et al., "Dedicated 3D photoacoustic breast imaging," Med. Phys. 40(11), 113301 (2013).
47. X. D. Wang et al., "Noninvasive laser-induced photoacoustic tomography for structural and functional in vivo imaging of the brain," Nat. Biotechnol. 21(7), 803-806 (2003).

48. J. Xia et al., "Whole-body ring-shaped confocal photoacoustic computed tomography of small animals in vivo," J. Biomed. Opt. 17(5), 050506 (2012).

49. C. H. Li and L. H. V. Wang, "Photoacoustic tomography of the mouse cerebral cortex with a high-numerical-aperture-based virtual point detector," J. Biomed. Opt. 14(2), 024047 (2009).

50. C. H. Li et al., "Real-time photoacoustic tomography of cortical hemodynamics in small animals," J. Biomed. Opt. 15(1), 010509 (2010).

51. A. Buehler et al., "Video rate optoacoustic tomography of mouse kidney perfusion," Opt. Lett. 35(14), 2475-2477 (2010).

52. L. M. Nie et al., "Photoacoustic tomography through a whole adult human skull with a photon recycler," J. Biomed. Opt. 17(11), 110506 (2012).

53. Y. Wang et al., "In vivo three-dimensional photoacoustic imaging based on a clinical matrix array ultrasound probe," J. Biomed. Opt. 17(6), 061208 (2012).

54. Y. Zhou et al., "Handheld photoacoustic probe to detect both melanoma depth and volume at high speed in vivo," J. Biophotonics 1(7) (2015).

55. E. Zhang, J. Laufer, and P. Beard, "Backward-mode multiwavelength photoacoustic scanner using a planar Fabry-Perot polymer film ultrasound sensor for high-resolution three-dimensional imaging of biological tissues," Appl. Opt. 47(4), 561-577 (2008).

56. D. Piras et al., "Photoacoustic imaging of the breast using the twente photoacoustic mammoscope: present status and future perspectives," IEEE J. Sel. Top. Quantum Electronics 16(4), 730-739 (2010).

57. M. Heijblom et al., "Visualizing breast cancer using the Twente photoacoustic mammoscope: What do we learn from twelve new patient measurements?" Opt. Express. 20(11), 11582-11597 (2012).

58. M. Heijblom et al., "Appearance of breast cysts in planar geometry photoacoustic mammography using 1064-nm excitation," J. Biomed. Opt. 18(12), 126009 (2013).

59. J. Laufer et al., "Three-dimensional noninvasive imaging of the vasculature in the mouse brain using a high resolution photoacoustic scanner," Appl. Optics 48(10), D299-D306 (2009).

60. J. Laufer et al., "In vivo preclinical photoacoustic imaging of tumor vasculature development and therapy," J. Biomed. Opt. 17(5), 056016 (2012).

61. J. Xia et al., "Wide-field two-dimensional multifocal optical-resolution photoacoustic-computed microscopy," Opt. Lett. 38(24), 5236-5239 (2013).

62. J. Laufer et al., "In vivo photoacoustic imaging of mouse embryos," J. Biomed. Opt. 17(6), 061220 (2012).

63. Y. Zhou, J. J. Yao, and L. H. V. Wang, "Optical clearing-aided photoacoustic microscopy with enhanced resolution and imaging depth," Opt. Lett. 38(14), 2592-2595 (2013).

64. Y. Liu, C. Zhang, and L. H. V. Wang, "Effects of light scattering on optical-resolution photoacoustic microscopy," J. Biomed. Opt. 17(12), 126014 (2012).

65. P. F. Hai et al., "Near-infrared optical-resolution photoacoustic microscopy," Opt. Lett. 39(17), 5192-5195 (2014).

66. S. Hu et al., "Label-free photoacoustic ophthalmic angiography," Opt. Lett. 35(1), 1-3 (2010).

67. N. Wu et al., "High-resolution dual-modality photoacoustic ocular imaging," Opt. Lett. 39(8), 2451-2454 (2014).

68. W. Song et al., "Integrating photoacoustic ophthalmoscopy with scanning laser ophthalmoscopy, optical coherence tomography, and fluorescein angiography for a multimodal retinal imaging platform," J. Biomed. Opt. 17(6), 061206 (2012).

69. X. J. Liu et al., "Optical coherence photoacoustic microscopy for in vivo multimodal retinal imaging," Opt. Lett. 40(7), 1370-1373 (2015).

70. J. J. Yao et al., "High-speed label-free functional photoacoustic microscopy of mouse brain in action," Nat. Methods 12(5), 407-410 (2015).

71. S. Hu et al., "Functional transcranial brain imaging by optical-resolution photoacoustic microscopy," J. Biomed. Opt. 14(4), 040503 (2009).

72. Y. Zhou et al., "Microcirculatory changes identified by photoacoustic microscopy in patients with complex regional pain syndrome type I after stellate ganglion blocks," J. Biomed. Opt. 19(8), 086017 (2014).

73. H. F. Zhang et al., "In vivo volumetric imaging of subcutaneous microvasculature by photoacoustic microscopy," Opt. Express. 14(20), 93179323 (2006). 
74. C. P. Favazza, L. A. Cornelius, and L. H. V. Wang, "In vivo functional photoacoustic microscopy of cutaneous microvasculature in human skin," J. Biomed. Opt. 16(2), 026004 (2011).

75. C. P. Favazza et al., "In vivo photoacoustic microscopy of human cutaneous microvasculature and a nevus," J. Biomed. Opt. 16(1), 016015 (2011).

76. C. Zhang, K. Maslov, and L. H. V. Wang, "Subwavelength-resolution label-free photoacoustic microscopy of optical absorption in vivo," Opt. Lett. 35(19), 3195-3197 (2010).

77. L. D. Wang et al., "Video-rate functional photoacoustic microscopy at depths," J. Biomed. Opt. 17(10), 106007 (2012).

78. H. X. Ke et al., "Performance characterization of an integrated ultrasound, photoacoustic, and thermoacoustic imaging system," $J$. Biomed. Opt. 17(5), 056010 (2012).

79. D. K. Yao et al., "In vivo label-free photoacoustic microscopy of cell nuclei by excitation of DNA and RNA," Opt. Lett. 35(24), 4139-4141 (2010).

80. D. K. Yao et al., "Optimal ultraviolet wavelength for in vivo photoacoustic imaging of cell nuclei," J. Biomed. Opt. 17(5), 056004 (2012).

81. C. Zhang et al., "Label-free photoacoustic microscopy of cytochromes," J. Biomed. Opt. 18(2), 020504 (2013).

82. Y. Zhou et al., "Photoacoustic microscopy of bilirubin in tissue phantoms," J. Biomed. Opt. 17(12), 126019 (2012).

83. C. Zhang et al., "Label-free photoacoustic microscopy of myocardial sheet architecture," J. Biomed. Opt. 17(6), 060506 (2012).

84. B. S. Goldschmidt et al., "Photoacoustic measurement of refractive index of dye solutions and myoglobin for biosensing applications," Biomed. Opt. Express. 4(11), 2463-2476 (2013).

85. H. F. Zhang et al., "Imaging of hemoglobin oxygen saturation variations in single vessels in vivo using photoacoustic microscopy," Appl. Phys. Lett. 90(5), 053901 (2007).

86. Y. Zhou et al., "Calibration-free absolute quantification of particle concentration by statistical analyses of photoacoustic signals in vivo," J. Biomed. Opt. 19(3), 037001 (2014).

87. M. Tang et al., "Noninvasive photoacoustic microscopy of methemoglobin in vivo," J. Biomed. Opt. 20(3), 036007 (2015).

88. Z. J. Chen, S. H. Yang, and D. Xing, "In vivo detection of hemoglobin oxygen saturation and carboxyhemoglobin saturation with multiwavelength photoacoustic microscopy," Opt. Lett. 37(16), 3414-3416 (2012).

89. J. Staley et al., "Growth of melanoma brain tumors monitored by photoacoustic microscopy," J. Biomed. Opt. 15(4), 040510 (2010).

90. Y. Zhou et al., "Handheld photoacoustic microscopy to detect melanoma depth in vivo," Opt. Lett. 39(16), 4731-4734 (2014).

91. H. W. Wang et al., "Label-free bond-selective imaging by listening to vibrationally excited molecules," Phys. Rev. Lett. 106(23), 238106 (2011).

92. V. V. Yakovlev et al., "Stimulated Raman photoacoustic imaging," Proc. Natl. Acad. Sci. U. S. A. 107(47), 20335-20339 (2010).

93. V. V. Yakovlev et al., "Monitoring stimulated Raman scattering with photoacoustic detection," Opt. Lett. 36(7), 1233-1235 (2011).

94. Z. Xu, Q. I. Zhu, and L. H. V. Wang, "In vivo photoacoustic tomography of mouse cerebral edema induced by cold injury," J. Biomed. Opt. 16(6), 066020 (2011).

95. Z. Xu, C. H. Li, and L. V. Wang, "Photoacoustic tomography of water in phantoms and tissue," J. Biomed. Opt. 15(3), 036019 (2010).

96. J. Kottmann et al., "Glucose sensing in human epidermis using midinfrared photoacoustic detection," Biomed. Opt. Express 3(4), 667-680 (2012).

97. M. A. Pleitez et al., "In vivo noninvasive monitoring of glucose concentration in human epidermis by mid-infrared pulsed photoacoustic spectroscopy," Anal. Chem. 85(2), 1013-1020 (2013).

98. P. Wang et al., "High-speed intravascular photoacoustic imaging of lipid-laden atherosclerotic plaque enabled by a $2-\mathrm{kHz}$ barium nitrite Raman laser," Sci. Rep. 4 (2014).

99. B. Wang et al., "Detection of lipid in atherosclerotic vessels using ultrasound-guided spectroscopic intravascular photoacoustic imaging," Opt. Express. 18(5), 4889-4897 (2010).

100. Y. Wang and R. K. Wang, "Photoacoustic recovery of an absolute optical absorption coefficient with an exact solution of a wave equation," Phys. Med. Biol. 53(21), 6167-6177 (2008).

101. Z. J. Guo et al., "Quantitative photoacoustic microscopy of optical absorption coefficients from acoustic spectra in the optical diffusive regime," J. Biomed. Opt. 17(6), 066011 (2012).
102. J. Xia et al., "Calibration-free quantification of absolute oxygen saturation based on the dynamics of photoacoustic signals," Opt. Lett. 38(15), 2800-2803 (2013).

103. Y. Y. Petrov et al., "Multiwavelength optoacoustic system for noninvasive monitoring of cerebral venous oxygenation: a pilot clinical test in the internal jugular vein," Opt. Lett. 31(12), 1827-1829 (2006).

104. S. Zackrisson, S. M. W. Y. van de Ven, and S. S. Gambhir, "Light in and sound out: emerging translational strategies for photoacoustic imaging," Cancer Res. 74(4), 979-1004 (2014).

105. G. P. Luke, D. Yeager, and S. Y. Emelianov, "Biomedical applications of photoacoustic imaging with exogenous contrast agents," Ann. Biomed. Eng. 40(2), 422-437 (2012).

106. A. De La Zerda et al., "Carbon nanotubes as photoacoustic molecular imaging agents in living mice," Nat. Nanotechnol. 3(9), 557-562 (2008).

107. C. Kim et al., "In vivo photoacoustic mapping of lymphatic systems with plasmon-resonant nanostars," J. Mater. Chem. 21(9), 2841-2844 (2011).

108. C. Kim et al., "In vivo molecular photoacoustic tomography of melanomas targeted by bioconjugated gold nanocages," ACS Nano 4(8), 4559-4564 (2010).

109. D. P. J. Pan et al., "Photoacoustic sentinel lymph node imaging with self-assembled copper neodecanoate nanoparticles," ACS Nano 6(2), 1260-1267 (2012).

110. J. J. Yao et al., "Evans blue dye-enhanced capillary-resolution photoacoustic microscopy in vivo," J. Biomed. Opt. 14(5), 054049 (2009).

111. S. Ashkenazi, "Photoacoustic lifetime imaging of dissolved oxygen using methylene blue," J. Biomed. Opt. 15(4), 040501 (2010).

112. X. D. Wang et al., "Noninvasive photoacoustic angiography of animal brains in vivo with near-infrared light and an optical contrast agent," Opt. Lett. 29(7), 730-732 (2004).

113. G. Ku and L. H. V. Wang, "Deeply penetrating photoacoustic tomography in biological tissues enhanced with an optical contrast agent," Opt. Lett. 30(5), 507-509 (2005).

114. M. R. Chatni et al., "Functional photoacoustic microscopy of $\mathrm{pH}$," J. Biomed. Opt. 16(10), 100503 (2011).

115. K. H. Song et al., "Noninvasive photoacoustic identification of sentinel lymph nodes containing methylene blue in vivo in a rat model," J. Biomed. Opt. 13(5), 054033 (2008).

116. L. Li et al., "Photoacoustic imaging of lacZ gene expression in vivo," J. Biomed. Opt. 12(2), 020504 (2007).

117. X. Cai et al., "Multi-scale molecular photoacoustic tomography of gene expression," PLoS One 7(8), e43999 (2012).

118. C. J. H. Ho et al., "Multifunctional photosensitizer-based contrast agents for photoacoustic imaging," Sci. Rep. 4 (2014).

119. A. B. Attia et al., "Phthalocyanine photosensitizers as contrast agents for in vivo photoacoustic tumor imaging," Biomed. Opt. Express 6(2), 591-598 (2015).

120. E. Morgounova et al., "Photoacoustic lifetime contrast between methylene blue monomers and self-quenched dimers as a model for dual-labeled activatable probes," J. Biomed. Opt. 18(5), 056004 (2013).

121. J. Yao et al., "Multiscale photoacoustic tomography using reversibly switchable bacterial phytochrome as a near-infrared photochromic probe," Nat Methods (2015).

122. A. C. Stiel et al., "High-contrast imaging of reversibly switchable fluorescent proteins via temporally unmixed multispectral optoacoustic tomography," Opt. Lett. 40(3), 367-370 (2015).

123. E. I. Galanzha et al., "Photoacoustic and photothermal cytometry using photoswitchable proteins and nanoparticles with ultrasharp resonances," J. Biophotonics 8(1-2), 81-93 (2015).

124. R. J. Zemp et al., "Realtime photoacoustic microscopy of murine cardiovascular dynamics," Opt. Express. 16(22), 18551-18556 (2008).

125. A. Taruttis et al., "Real-time imaging of cardiovascular dynamics and circulating gold nanorods with multispectral optoacoustic tomography," Opt. Express. 18(19), 19592-19602 (2010).

126. L. A. Song et al., "Ultrasound-array-based real-time photoacoustic microscopy of human pulsatile dynamics in vivo," J. Biomed. Opt. 15(2), 021303 (2010).

127. K. Aizawa et al., "Photoacoustic monitoring of burn healing process in rats," J. Biomed. Opt. 13(6), 064020 (2008).

128. S. Sato et al., "Photoacoustic diagnosis of burns in rats," J. Trauma 59(6), 1450-1455 (2005).

129. H. F. Zhang et al., "Imaging acute thermal burns by photoacoustic microscopy," J. Biomed. Opt. 11(5), 054033 (2006). 
130. A. de la Zerda et al., "Ultrahigh sensitivity carbon nanotube agents for photoacoustic molecular imaging in living mice," Nano Lett. 10(6), 2168-2172 (2010).

131. M. L. Li et al., "In-vivo photoacoustic microscopy of nanoshell extravasation from solid tumor vasculature," J. Biomed. Opt. 14(1), 010507 (2009).

132. J. Kang et al., "Photoacoustic imaging of breast microcalcifications: a validation study with 3-dimensional ex vivo data and spectrophotometric measurement," J. Biophotonics 8(1-2), 71-80 (2015).

133. C. Sim et al., "Photoacoustic-based nanomedicine for cancer diagnosis and therapy," J. Control Release 203, 118-125 (2015).

134. S. Y. Nam and S. Y. Emelianov, "Array-based real-time ultrasound and photoacoustic ocular imaging," J. Opt. Soc. Korea 18(2), 151-155 (2014).

135. W. Song et al., "Multimodal photoacoustic ophthalmoscopy in mouse," J. Biophotonics 6(6-7), 505-512 (2013).

136. T. Liu et al., "Fundus camera guided photoacoustic ophthalmoscopy," Curr. Eye Res. 38(12), 1229-1234 (2013).

137. J. M. Yang et al., "Three-dimensional photoacoustic endoscopic imaging of the rabbit esophagus," PLoS One 10(4) (2015).

138. P. Hajireza, W. Shi, and R. Zemp, "Label-free in vivo GRIN-lens optical resolution photoacoustic micro-endoscopy," Laser Phys. Lett. 10(5), 055603 (2013).

139. P. Hajireza et al., "Optical resolution photoacoustic microendoscopy with ultrasound-guided insertion and array system detection," J. Biomed. Opt. 18(9), 090502 (2013).

140. J. M. Yang et al., "A 2.5 -mm diameter probe for photoacoustic and ultrasonic endoscopy," Opt. Express 20(21), 23944-23953 (2012).

141. M. Kneipp et al., "Functional real-time optoacoustic imaging of middle cerebral artery occlusion in mice," PLoS One 9(4) (2014).

142. X. S. Bai et al., "Intravascular optical-resolution photoacoustic tomography with a $1.1 \mathrm{~mm}$ diameter catheter," PLoS One 9(3) (2014).

143. E. I. Galanzha et al., "In vivo flow cytometry of circulating clots using negative photothermal and photoacoustic contrasts," Cytom Part A 79A(10), 814-824 (2011).

144. L. D. Liao et al., "Transcranial imaging of functional cerebral hemodynamic changes in single blood vessels using in vivo photoacoustic microscopy," J. Cereb. Blood Flow Metab. 32(6), 938-951 (2012).

145. L. D. Liao et al., "Imaging brain hemodynamic changes during rat forepaw electrical stimulation using functional photoacoustic microscopy," NeuroImage 52(2), 562-570 (2010).

146. J. J. Yao et al., "Noninvasive photoacoustic computed tomography of mouse brain metabolism in vivo," NeuroImage 64, 257-266 (2013).

147. V. Tsytsarev et al., "Photoacoustic microscopy of microvascular responses to cortical electrical stimulation," J. Biomed. Opt. 16(7), 076002 (2011).

148. M. Heijblom, W. Steenbergen, and S. Manohar, "Clinical photoacoustic breast imaging," IEEE Pulse 6(3), 42-46 (2015).

149. T. Gould, Q. Z. Wang, and T. J. Pfefer, "Optical-thermal light-tissue interactions during photoacoustic breast imaging," Biomed. Opt. Express 5(3), 832-847 (2014).

150. W. F. Xia et al., "An optimized ultrasound detector for photoacoustic breast tomography," Med. Phys. 40(3), 032901 (2013).

151. L. Lin et al., "In vivo deep brain imaging of rats using oral-cavity illuminated photoacoustic computed tomography," J. Biomed. Opt. 20(1), 016019 (2015).

152. M. Nasiriavanaki et al., "High-resolution photoacoustic tomography of resting-state functional connectivity in the mouse brain," Proc. Natl. Acad. Sci. U. S. A. 111(1), 21-26 (2014).

153. R. Y. Zhang et al., "In vivo optically encoded photoacoustic flowgraphy," Opt. Lett. 39(13), 3814-3817 (2014).

154. C. Zhang et al., "Slow-sound photoacoustic microscopy," Appl. Phys. Lett. 102(16) (2013).

155. C. Zhang et al., "Reflection-mode submicron-resolution in vivo photoacoustic microscopy," J. Biomed. Opt. 17(2), 020501 (2012).

156. J. J. Yao et al., "Double-illumination photoacoustic microscopy," Opt. Lett. 37(4), 659-661 (2012).

157. X. T. Zou et al., "Polydimethylsiloxane thin film characterization using all-optical photoacoustic mechanism," Appl. Opt. 52(25), 6239-6244 (2013).

158. E. Z. Zhang et al., "In vivo high-resolution 3D photoacoustic imaging of superficial vascular anatomy," Phys. Med. Biol. 54(4), 1035-1046 (2009).
159. Y. Zhou et al., "Calibration-free in vivo transverse blood flowmetry based on cross correlation of slow time profiles from photoacoustic microscopy," Opt. Lett. 38(19), 3882-3885 (2013).

160. J. J. Yao et al., "Absolute photoacoustic thermometry in deep tissue," Opt. Lett. 38(24), 5228-5231 (2013).

161. J. Y. Liang et al., "Cross-correlation-based transverse flow measurements using optical resolution photoacoustic microscopy with a digital micromirror device," J. Biomed. Opt. 18(9), 096004 (2013).

162. J. J. Yao et al., "Label-free oxygen-metabolic photoacoustic microscopy in vivo," J. Biomed. Opt. 16(7), 076003 (2011).

163. B. Ning et al., "Ultrasound-aided multi-parametric photoacoustic microscopy of the mouse brain," Sci. Rep. 5, 18775 (2015).

164. G. Li et al., "Multiview Hilbert transformation for full-view photoacoustic computed tomography using a linear array," J. Biomed. Opt. 20(6), 066010 (2015).

165. J. Xia et al., "Retrospective respiration-gated whole-body photoacoustic computed tomography of mice," J. Biomed. Opt. 19(1), 016003 (2014).

166. S. N. Hennen et al., "Photoacoustic tomography imaging and estimation of oxygen saturation of hemoglobin in ocular tissue of rabbits," Exp. Eye Res. 138, 153-158 (2015).

167. L. D. Wang, K. Maslov, and L. H. V. Wang, "Single-cell label-free photoacoustic flowoxigraphy in vivo," Proc. Natl. Acad. Sci. U. S. A. 110(15), 5759-5764 (2013).

168. A. Garcia-Uribe et al., "Dual-modality photoacoustic and ultrasound imaging system for noninvasive sentinel lymph node detection in patients with breast cancer," Sci. Rep. 5, 15748 (2015).

169. A. Danielli et al., "Label-free photoacoustic nanoscopy," J. Biomed. Opt. 19(8), 086006 (2014).

170. Y. Zhou, J. Liang, and L. V. Wang, "Cuffing-based photoacoustic flowmetry in humans in the optical diffusive regime," J. Biophotonics (2015).

171. Y. Zhou et al., "In vivo photoacoustic flowmetry at depths of the diffusive regime based on saline injection," J. Biomed. Opt. 20(8), 087001 (2015).

172. C. H. Yeh et al., "Photoacoustic microscopy of blood pulse wave," J. Biomed. Opt. 17(7), 070504 (2012).

173. X. H. Zhu et al., "Simultaneous and noninvasive imaging of cerebral oxygen metabolic rate, blood flow and oxygen extraction fraction in stroke mice," NeuroImage 64, 437-447 (2013).

174. A. Krumholz et al., "Functional photoacoustic microscopy of diabetic vasculature," J. Biomed. Opt. 17(6), 060502 (2012).

175. D. Yeager et al., "Intravascular photoacoustic imaging of exogenously labeled atherosclerotic plaque through luminal blood," J. Biomed. Opt. 17(10), 106016 (2012).

176. M. Wu et al., "Impact of device geometry on the imaging characteristics of an intravascular photoacoustic catheter," Appl. Opt. 53(34), 8131-8139 (2014).

177. B. Wang et al., "Photoacoustic tomography system for noninvasive real-time three-dimensional imaging of epilepsy," Biomed. Opt. Express 3(6), 1427-1432 (2012).

Yong Zhou is currently a graduate student in biomedical engineering at Washington University in St. Louis, under the supervision of Dr. Lihong V. Wang, Gene K. Beare Distinguished Professor. His research focuses on the development of photoacoustic imaging systems.

Junjie Yao received his BE and ME degrees in biomedical engineering from Tsinghua University, Beijing, in 2006 and 2008, respectively, under the tutelage of Prof. Jing Bai. He received his $\mathrm{PhD}$ in biomedical engineering at Washington University in St. Louis (WUSTL), in 2013, under the tutelage of Prof. Lihong V. Wang. He is currently a postdoctoral research associate at WUSTL. His research interest is in photoacoustic, optical, and ultrasound imaging technologies in biomedicine.

Lihong V. Wang is the Beare distinguished professor at Washington University, has published 425 journal articles (h-index $=96$, citations $>36,000$ ) and delivered 420 keynote/plenary/invited talks. His laboratory published the first functional photoacoustic CT and 3-D photoacoustic microscopy. He received the Goodman Award for his Biomedical Optics textbook, NIH Director's Pioneer Award, OSA Mees Medal, IEEE Technical Achievement and Biomedical Engineering Awards, SPIE Britton Chance Biomedical Optics Award, and an honorary doctorate from Lund University, Sweden. 